quatrième série-tome 43 fascicule 6 novembre-décembre 2010

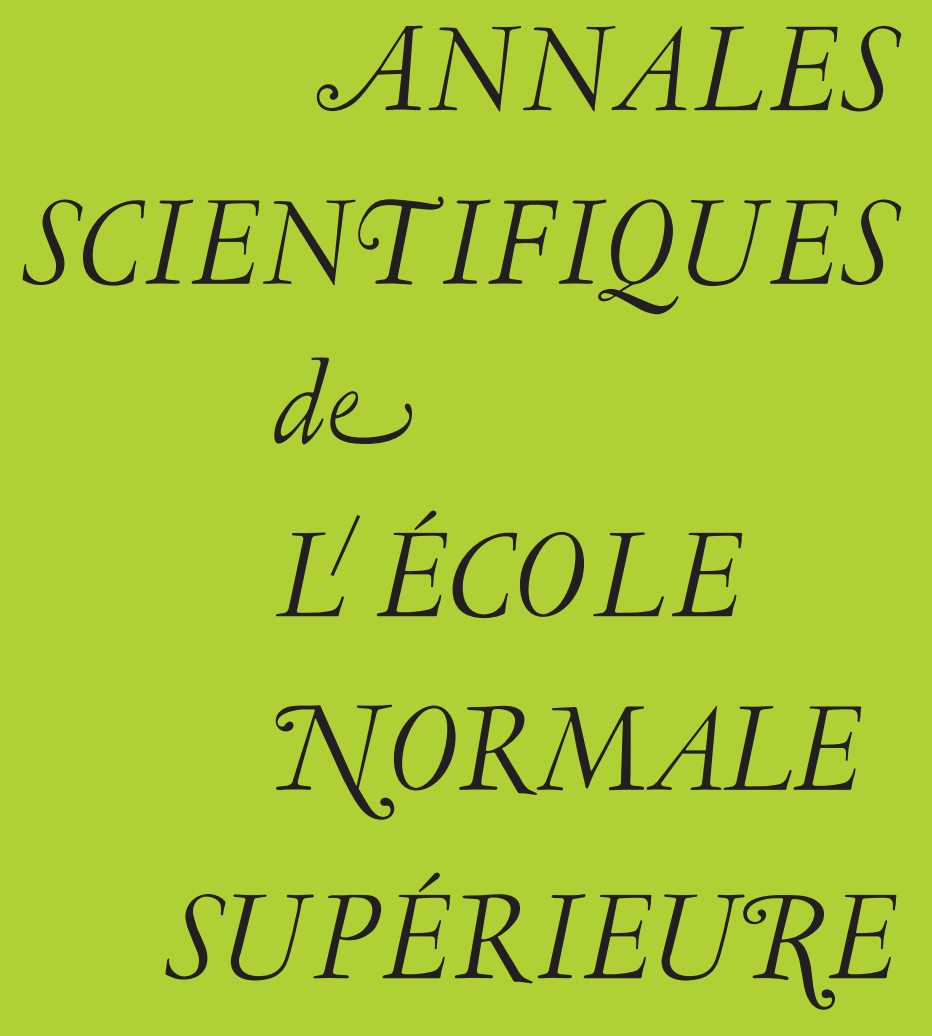

Marc-Hubert NICOLE \& Adrian VASIU \& Torsten WEDHORN Purity of level $m$ stratifications 
Ann. Scient. Éc. Norm. Sup.

$4^{\text {e }}$ série, t. 43, 2010, p. 925 à 955

\title{
PURITY OF LEVEL $m$ STRATIFICATIONS
}

\author{
By Marc-Hubert Nicole, Adrian VASiU \\ AND TORSTEN WEDHORN
}

\begin{abstract}
Let $k$ be a field of characteristic $p>0$. Let $D_{m}$ be a BT $m$ over $k$ (i.e., an $m$-truncated Barsotti-Tate group over $k$ ). Let $S$ be a $k$-scheme and let $X$ be a $\mathrm{BT}_{m}$ over $S$. Let $S_{D_{m}}(X)$ be the subscheme of $S$ which describes the locus where $X$ is locally for the fppf topology isomorphic to $D_{m}$. If $p \geq 5$, we show that $S_{D_{m}}(X)$ is pure in $S$, i.e. the immersion $S_{D_{m}}(X) \hookrightarrow S$ is affine. For $p \in\{2,3\}$, we prove purity if $D_{m}$ satisfies a certain technical property depending only on its $p$-torsion $D_{m}[p]$. For $p \geq 5$, we apply the developed techniques to show that all level $m$ stratifications associated to Shimura varieties of Hodge type are pure.

RÉSUMÉ. - Soit $k$ un corps de caractéristique $p>0$. Soit $D_{m}$ un $\mathrm{BT}_{m}$ sur $k$ (i.e., un groupe de Barsotti-Tate tronqué en échelon $m$ sur $k$ ). Soient $S$ un $k$-schéma et $X$ un $\mathrm{BT}_{m}$ sur $S$. Soit $S_{D_{m}}(X)$ le sous-schéma de $S$ correspondant au lieu où $X$ est isomorphe à $D_{m}$ localement pour la topologie fppf. Si $p \geq 5$, nous montrons que $S_{D_{m}}(X)$ est pur dans $S$, i.e. l'immersion $S_{D_{m}}(X) \hookrightarrow S$ est affine. Pour $p \in\{2,3\}$, nous prouvons la pureté pour $D_{m}$ satisfaisant une certaine propriété technique dépendant uniquement de la $p$-torsion $D_{m}[p]$. Pour $p \geq 5$, nous utilisons les techniques développées pour montrer que toutes les stratifications par l'échelon associées aux variétés de Shimura de type Hodge sont pures.
\end{abstract}

\section{Introduction}

Let $p$ be a prime number. Let $k$ be a field of characteristic $p$. Let $c, d$, and $m$ be positive integers. In this paper, a $\mathrm{BT}_{m}$ is an $m$-truncated Barsotti-Tate group of codimension $c$ and dimension $d$. Let $D_{m}$ be a fixed $\mathrm{BT}_{m}$ over $k$.

Let $S$ be an arbitrary $k$-scheme and let $X_{m}$ be a $\mathrm{BT}_{m}$ over $S$. Let $S_{D_{m}}\left(X_{m}\right)$ be the (necessarily unique) locally closed subscheme of $S$ that satisfies the following property. A morphism $f: S^{\prime} \rightarrow S$ of $k$-schemes factors through $S_{D_{m}}\left(X_{m}\right)$ if and only if $f^{*}\left(X_{m}\right)$ and $D_{m} \times_{\text {Spec } k} S^{\prime}$ are locally for the fppf topology isomorphic as $\mathrm{BT}_{m}$ 's over $S^{\prime}$ (see Subsection 2.2 for the existence of $S_{D_{m}}\left(X_{m}\right)$ ). If $D$ is either a $\mathrm{BT}_{m^{\prime}}$ for some $m^{\prime} \geq m$, or a $p$-divisible group over $k$, we will also write $S_{D}\left(X_{m}\right)$ instead of $S_{D\left[p^{m}\right]}\left(X_{m}\right)$.

The following notion of purity (that has already been considered in [24], Section 2.1.1) will be central. 
Definition 1.1. - A subscheme $T$ of a scheme $S$ is called pure in $S$ if the immersion $T \hookrightarrow S$ is affine.

We remark that the purity of $T$ in a locally noetherian scheme $S$ implies the following weaker variant of purity: If $Y$ is an irreducible component of the Zariski closure $\bar{T}$ of $T$ in $S$, then the complement of $Y \cap T$ in $Y$ is either empty or of pure codimension 1. On the other hand, if $S$ is separated and $T$ is (globally) an affine scheme, then $T$ is pure in $S$.

Purity results for strata defined by $p$-divisible groups have a long history. The earliest hints of purity are probably the computations mentioned by Y. Manin in [15], at the bottom of p. 44. For Newton polygon strata, J. de Jong and F. Oort have shown the above mentioned weaker version of purity in [11] and one of us has shown in [24] that these strata are even pure in the sense of Definition 1.1. For $p$-rank strata, Th. Zink proved in [29] the weaker version of purity. Moreover, T. Itō proved in [10] the existence of generalized Hasse-Witt invariants for PEL unitary Shimura varieties of signature $(n-1,1)$ at primes $p$ where the unitary group is split. This result implies in fact a stronger kind of purity (see below).

The weak version of purity is an important tool to estimate and compute the dimensions of strata in the locally noetherian case. Purity itself is an important step towards determining whether a (quasi-affine) stratum is in fact affine, or whether a cohomological sheaf is in fact zero. For instance, a genuine (cohomological) application of purity (and not of global affineness!) to some simple Shimura varieties can be found in [21], Proposition 6.2.

The goal of this paper is to show that $S_{D_{m}}\left(X_{m}\right)$ is pure for all schemes $S$ and all $B T_{m}$ 's $X_{m}$ if $D_{m}$ satisfies a certain condition (C) introduced in Subsection 4.2. Here we remark that condition (C) depends only on $D_{m}[p]$ and it can be checked easily. Condition $(\mathrm{C})$ is satisfied if any one of the three conditions below holds (cf. Lemma 4.3 (c) and (d) and Example 4.4):

(i) We have $p \geq 5$.

(ii) We have $p=3$ and $\min (c, d) \leq 6$.

(iii) There exists an integer $a \geq 2$ such that we have a ring monomorphism $\mathbb{F}_{p^{a}} \hookrightarrow \operatorname{End}\left(D_{m}[p]\right)$ with the property that $\mathbb{F}_{p^{a}}$ acts on the tangent space of $D_{m}[p]$ via scalar endomorphisms.

For the remainder of the introduction, we will assume that condition (C) holds for $D_{m}$. The main result of the paper is the following theorem.

Theorem 1.2. - The locally closed subscheme $S_{D_{m}}\left(X_{m}\right)$ is pure in $S$.

We obtain the following corollary.

COROLlary 1.3. - Let $S$ be locally noetherian and let $Y$ be an irreducible component of $\overline{S_{D_{m}}\left(X_{m}\right)}$. Then the complement of $S_{D_{m}}\left(X_{m}\right) \cap Y$ in $Y$ is either empty or of pure codimension 1 .

Now let $D$ be a $p$-divisible group over $k$ such that $D\left[p^{m}\right]=D_{m}$. For every reduced $k$-scheme $S$ and every $p$-divisible group $X$ over $S$ denote by $\mathfrak{n}_{D}(X)$ the (necessarily unique) reduced locally closed subscheme of $S$ such that for each field extension $K$ of $k$ we have

$$
\mathfrak{n}_{D}(X)(K)=\left\{s \in S(K) \mid D \text { and } s^{*}(X) \text { have equal Newton polygons }\right\} .
$$

$4^{\text {e }}$ SÉRIE - TOME $43-2010-\mathrm{N}^{\mathrm{o}} 6$ 
Thus $\mathfrak{n}_{D}(X)$ is the Newton polygon stratum of $S$ defined by $X$ that corresponds to the Newton polygon of $D$. The locally closed subscheme $\mathfrak{n}_{D}(X)$ is pure in $S$ by [24], Theorem 1.6. Thus we get another purity result:

Corollary 1.4. - For each $m \in \mathbb{N}^{*}$, the locally closed subscheme $\mathfrak{n}_{D}(X) \cap S_{D}\left(X\left[p^{m}\right]\right)$ is pure in $S$.

Moreover, we can use the well known fact that there exists an integer $n_{D} \geq 1$ with the following property. If $C$ is a $p$-divisible group over an algebraic closure $\bar{k}$ of $k$ such that $C\left[p^{n_{D}}\right]$ is isomorphic to $D\left[p^{n_{D}}\right]_{\bar{k}}$, then $C$ is isomorphic to $D_{\bar{k}}$ (for instance, see [22], Theorem 1 or [24], Corollary 1.3). We assume that $n_{D}$ is chosen minimal. Then there exists a (necessarily unique) reduced locally closed subscheme $\mathfrak{u}_{D}(X)$ of $S$ such that for every algebraically closed field extension $K$ of $k$ we have

$$
\mathfrak{u}_{D}(X)(K)=\left\{s \in S(K) \mid D_{K} \cong s^{*}(X)\right\} .
$$

Indeed, we have $\mathfrak{u}_{D}(X)=S_{D}\left(X\left[p^{n_{D}}\right]\right)_{\text {red }}$. From Theorem 1.2, we obtain the following purity result:

Corollary 1.5. - The locally closed subscheme $\mathfrak{u}_{D}(X)$ is pure in $S$.

For special fibres of good integral models in unramified mixed characteristic $(0, p)$ of Shimura varieties of Hodge type (or more generally, for quasi Shimura $p$-varieties of Hodge type), there exists a level $m$ stratification that parametrizes $\mathrm{BT}_{m}$ 's with additional structures (see Subsection 6.2). The proof of Theorem 1.2 can be adapted to show that all level $m$ stratifications are pure (see Theorem 6.3), provided they are either in characteristic $p \geq 5$ or are in characteristic $p \in\{2,3\}$ and an additional condition holds.

In this introduction, we will only state the Siegel modular varieties variant of Theorem 1.2 (see Example 6.5). Let $N \geq 3$ be an integer prime to $p$. Let $\mathscr{A}_{d, 1, N}$ be the Mumford moduli scheme that parameterizes principally polarized abelian schemes over $\mathbb{F}_{p}$-schemes of relative dimension $d$ and equipped with a symplectic similitude level $N$ structure (cf. [18], Theorems 7.9 and 7.10). Let $(\mathscr{U}, \Lambda)$ be the principally quasi-polarized $p$-divisible group of the universal principally polarized abelian scheme over $\mathscr{A}_{d, 1, N}$. If $k$ is algebraically closed and if $(D, \lambda)$ is a principally quasi-polarized $p$-divisible group of height $2 d$ over $k$, let $\mathfrak{s}_{D, \lambda}(m)$ be the unique reduced locally closed subscheme of $\mathscr{A}_{d, 1, N, k}$ that satisfies the following identity of sets

$$
\mathfrak{s}_{D, \lambda}(m)(k)=\left\{y \in \mathscr{A}_{d, 1, N}(k) \mid y^{*}(\mathscr{U}, \Lambda)\left[p^{m}\right] \cong(D, \lambda)\left[p^{m}\right]\right\} .
$$

Then $\mathfrak{s}_{D, \lambda}(m)$ is regular and equidimensional (see [25], Corollary 4.3 and Example 4.5; Subsection 2.3 below can be easily adapted to prove the existence and the smoothness of the $k$-scheme $\left.\mathfrak{s}_{D, \lambda}(m)\right)$. Moreover we have:

Theorem 1.6. - If either $p=3$ and $d \leq 6$ or $p \geq 5$, then the locally closed subscheme $\mathfrak{s}_{D, \lambda}(m)$ is pure in $\mathscr{A}_{d, 1, N, k}$.

We remark that for $m=1$, Theorem 1.6 neither implies nor is implied by Oort's result ([20], Theorem 1.2) which asserts (for all primes $p$ ) that the scheme $\mathfrak{s}_{D, \lambda}(1)$ is quasi-affine.

Finally, we investigate briefly the following stronger notion of purity. 
DeFINITION 1.7. - Let $T \rightarrow S$ be a quasi-compact immersion and let $\bar{T}$ be the schemetheoretic closure of $T$ in $S$. Then $T$ is called Zariski locally principally pure in $S$ if locally for the Zariski topology of $\bar{T}$, there exists a function $f \in \Gamma\left(\bar{T}, \mathscr{O}_{\bar{T}}\right)$ such that we have $T=\bar{T}_{f}$, where $\bar{T}_{f}$ is the largest open subscheme of $\bar{T}$ over which $f$ is invertible.

We obtain variants of this notion by replacing the Zariski topology by another Grothendieck topology $\mathscr{T}$ of $S$. If $\mathscr{T}$ is coarser than the fpqc topology (e.g., the Zariski or the étale topology), each $\mathscr{T}$ locally principally pure subscheme is pure (as affineness for morphisms is a local property for the fpqc topology). Principal purity for $p$-rank strata corresponds to the existence of generalized Hasse-Witt invariants. They have been investigated by T. Itō for certain unitary Shimura varieties (see [10]) and by E. Z. Goren for Hilbert modular varieties (see [4]).

In Section 7, we will show that this stronger notion of purity does not hold in general. In fact, we have:

Proposition 1.8. - Let $c, d \geq 2$ and $s \in\{1, \ldots, c-1\}$. Then the strata of p-rank equal to $s$ associated to $B T_{1}$ 's over $\mathbb{F}_{p}$-schemes of codimension $c$ and dimension $d$, are not étale locally principally pure in general.

We now give an overview of the structure of the paper. In Section 2, we define the level $m$ strata $S_{D_{m}}\left(X_{m}\right)$ and we prove some basic properties of them. Then we make a dévissage to the following situation.

Essential Situation 1.9. - Let $k$ be an algebraically closed field of characteristic $p>0$ and let $D_{m}$ be a $\mathrm{BT}_{m}$ over $k$ which satisfies condition (C). Let $D$ be a $p$-divisible group over $k$ such that $D\left[p^{m}\right]=D_{m}$. Let $S=\mathscr{A}$ be a smooth $k$-scheme of finite type which is equidimensional of dimension $c d$ and for which the following two properties hold:

(a) There exists a $p$-divisible group $\mathscr{E}$ of codimension $c$ and dimension $d$ over $\mathscr{A}$ which is a versal deformation at each $k$-valued point of $\mathscr{A}$.

(b) There exists a point $y_{D} \in \mathscr{A}(k)$ such that $y_{D}^{*}(\mathscr{E})$ is isomorphic to $D$.

In this case we simply write $\mathfrak{s}_{D}(m)$ instead of $\mathscr{A}_{D}\left(\mathscr{E}\left[p^{m}\right]\right)$. In Subsection 2.3, we will prove that $\mathfrak{s}_{D}(m)$ is smooth over $k$ (by [25], Theorem 1.2 (a) and (b) and Remark 3.1.2 we know already that the reduced scheme of $\mathfrak{s}_{D}(m)$ is a smooth equidimensional $k$-scheme, although this fact is not used in the proof below). Then we show that Theorem 1.2 follows if $\mathfrak{s}_{D}(m)$ is pure in $\mathscr{A}$.

We remark that for $m \geq n_{D}$ (where $n_{D}$ is the integer defined above before Corollary 1.5) the fact that $\mathfrak{s}_{D}(m)$ is pure in $\mathscr{A}$ is proved in [24], Theorem 5.3.1 (c). This result of [24] and thus Corollary 1.5 also, hold even if condition (C) does not hold for $D[p]$.

The proof of Theorem 1.2 is presented in Section 5. There we show that purity follows from the affineness of a certain orbit $\mathscr{O}_{m}$ of a group action

$$
\mathbb{T}_{m}: \mathscr{H}_{m} \times \mathscr{D}_{m} \rightarrow \mathscr{D}_{m},
$$

which was introduced in [25]. The orbits of $\mathbb{T}_{m}$ parameterize isomorphism classes of $\mathrm{BT}_{m}$ 's over perfect fields. In fact we show that $\mathscr{O}_{m}$ is affine for all $m$ provided $\mathscr{O}_{1}$ is affine.

The definition of the action $\mathbb{T}_{m}$ is recalled in Section 3 , and in Section 4 the main properties of the action $\mathbb{T}_{1}$ we need are presented. There the condition $(C)$ is introduced and used. 
Its key role is to imply that a certain morphism between affine $k$-schemes is finite (see Theorem 4.8) which allows us in Subsection 5.2 to use Chevalley's theorem to show that $\mathscr{O}_{1}$ is affine.

In Section 6, we prove purity of the level $m$ stratification for quasi Shimura $p$-varieties of Hodge type. Finally, in Section 7, we show that generalized Hasse-Witt invariants do not always exist for Shimura varieties. Indeed, we construct a counterexample and we deduce Proposition 1.8.

\section{Dévissage to the Essential Situation}

\subsection{Moduli spaces of truncated Barsotti-Tate groups}

We recall that $c, d$, and $m$ are positive integers. Let $\mathscr{B} \mathscr{T}_{m}=\mathscr{B} \mathscr{T}_{m}^{c, d}$ be the moduli space of $m$-truncated Barsotti-Tate groups in characteristic $p$ that have codimension $c$ and dimension $d$. In other words, for each $\mathbb{F}_{p}$-scheme $S, \mathscr{B} \mathscr{T}_{m}(S)$ is the category of all $\mathrm{BT}_{m}$ 's over $S$ of codimension $c$ and dimension $d$, the morphisms in $\mathscr{B}_{m}(S)$ being isomorphisms of $\mathrm{BT}_{m}$ 's.

As explained in [28], Proposition (1.8) and Corollary (3.3), it follows from results of Illusie and Grothendieck (see [9], Théorème 4.4) that $\mathscr{B} \mathscr{T}_{m}$ is a smooth algebraic stack of finite type over $\mathbb{F}_{p}$. More precisely, $\mathscr{B} \mathscr{T}_{m}$ is an algebraic stack of the form $\left[\mathbf{G L}_{p^{m(c+d)}} \backslash Z_{m}\right]$, where $Z_{m}$ is a smooth quasi-affine $\mathbb{F}_{p}$-scheme on which $\mathbf{G L}_{p^{m(c+d)}}$ acts. Moreover, the canonical morphism $P: Z_{m} \rightarrow \mathscr{B} \mathscr{T}_{m}$ is a $\mathbf{G L}_{p^{m(c+d)}}$-torsor for the Zariski topology. Thus $P(R): Z_{m}(R) \rightarrow \mathscr{B} \mathscr{T}_{m}(R)$ is surjective for each commutative local $\mathbb{F}_{p}$-algebra $R$.

\subsection{The level $m$ stratification}

Let $k$ be a field of characteristic $p$ and let $D_{m}$ be a $\mathrm{BT}_{m}$ over $k$. By the definition of the stack $\mathscr{B} \mathscr{T}_{m}, D_{m}$ defines a 1-morphism over $k$

$$
\xi:=\xi_{D_{m}}: \operatorname{Spec} k \rightarrow \mathscr{B} \mathscr{T}_{m} \otimes_{\mathbb{F}_{p}} k
$$

The pair $(\xi, k)$ defines a point of $\mathscr{B} \mathscr{T}_{m} \otimes_{\mathbb{F}_{p}} k$ (in the sense of [14], Section 5.2) which we also denote by $\xi$. As $\mathscr{B} \mathscr{T}_{m}$ is locally noetherian, $\xi$ is algebraic by [14], Section 11.3 and its residue field is $k$. Let $\mathscr{G}_{\xi}$ be the residue gerbe of the point $\xi$; it is an algebraic stack which is an fppf gerbe over Spec $k$.

Lemma 2.1. - The canonical monomorphism $\mathscr{G}_{\xi} \rightarrow \mathscr{B}_{m} \otimes_{\mathbb{F}_{p}} k$ is representable by an immersion of finite presentation. The algebraic stack $\mathscr{G}_{\xi}$ is smooth over $\mathrm{Spec} k$.

Proof. - The morphism $\mathscr{G}_{\xi} \rightarrow \mathscr{B} \mathscr{T}_{m} \otimes_{\mathbb{F}_{p}} k$ is representable because $\xi$ is algebraic. For the remaining assertions we may assume that $k$ is algebraically closed. With the notations of Subsection 2.1, from [14], Exemple (11.2.2) we get that the fibre product of the diagram

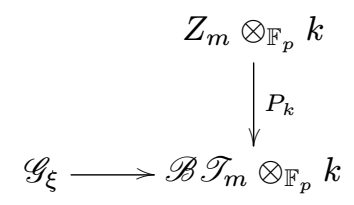




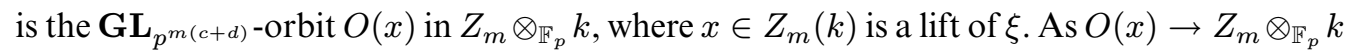
is a quasi-compact immersion of noetherian schemes, $\mathscr{G}_{\xi} \rightarrow \mathscr{B} \mathscr{T}_{m} \otimes_{\mathbb{F}_{p}} k$ is representable by an immersion of finite presentation.

The morphism $P_{k}$ is smooth and surjective and thus $O(x) \rightarrow \mathscr{G}_{\xi}$ is smooth and surjective. As $O(x)$ is smooth over $k, \mathscr{G}_{\xi}$ is smooth over $\operatorname{Spec} k$.

Let $S$ be an arbitrary $k$-scheme. Let $X_{m}$ be a $\mathrm{BT}_{m}$ over $S$ defining a 1-morphism $\xi_{X_{m}}: S \rightarrow \mathscr{B} \mathscr{T}_{m} \otimes_{\mathbb{F}_{p}} k$. Let $S_{D_{m}}\left(X_{m}\right)$ be the fibre product of the diagram

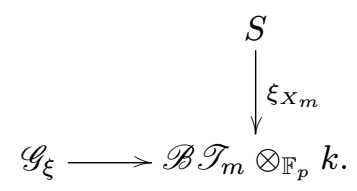

The canonical morphism $S_{D_{m}}\left(X_{m}\right) \rightarrow S$ is an immersion of finite presentation by Lemma 2.1. Thus we will view $S_{D_{m}}\left(X_{m}\right)$ as a locally closed subscheme of $S$. As $S_{D_{m}}\left(X_{m}\right) \rightarrow S$ is quasi-compact, its scheme-theoretic closure $\overline{S_{D_{m}}\left(X_{m}\right)}$ exists by [7], Corollaire (6.10.6).

By [14], Section (11.1), a morphism $f: S^{\prime} \rightarrow S$ of $k$-schemes factors through $S_{D_{m}}\left(X_{m}\right)$ if and only if $f^{*}\left(X_{m}\right)$ and $D_{m} \times_{\text {Spec } k} S^{\prime}$ are locally for the fppf topology isomorphic as $\mathrm{BT}_{m}$ 's over $S^{\prime}$. We call $S_{D_{m}}\left(X_{m}\right)$ the level $m$ stratum of $\left(S, X_{m}\right)$ with respect to $D_{m}$.

We note that the level 1 strata are the Ekedahl-Oort strata introduced in [20] and that the level $m$ strata were studied first in [28] and in [25].

\subsection{Dévissage}

We will show that it suffices to prove Theorem 1.2 in the Essential Situation 1.9. Generalizing Definition 1.1, we say that a substack $\mathscr{T}$ of an algebraic stack $\mathscr{S}$ is pure in $\mathscr{S}$ if the immersion $\mathscr{T} \hookrightarrow \mathscr{S}$ is affine. We recall the following lemma (which follows from the fact that the affineness property for a morphism is local for the fpqc topology, see [6], Proposition (2.7.1)).

Lemma 2.2. - Let $\mathscr{S}$ be an algebraic stack and let $\mathscr{T} \subset \mathscr{S}$ be a substack. Let $f: \mathscr{Y} \rightarrow \mathscr{S}$ be a representable morphism of algebraic stacks. We have the following two properties:

(1) If $\mathscr{T}$ is pure in $\mathscr{S}, f^{-1}(\mathscr{T})$ is pure in $\mathscr{Y}$.

(2) Conversely, assume that $f$ is quasi-compact and faithfully flat. If $f^{-1}(\mathscr{T})$ is pure in $\mathscr{Y}$, then $\mathscr{T}$ is pure in $\mathscr{S}$.

We now refer to the general situation of Subsection 2.2. It follows from the construction of $S_{D_{m}}\left(X_{m}\right)$ that to prove that the immersion $S_{D_{m}}\left(X_{m}\right) \hookrightarrow S$ is affine, it suffices to show that the immersion $\mathscr{G}_{\xi} \rightarrow \mathscr{B} \mathscr{T}_{m}$ is affine.

A smooth scheme $\mathscr{A}$ over $k$ of dimension $c d$ with a $p$-divisible group $\mathscr{E}$ over $\mathscr{A}$ satisfies the properties (a) and (b) of the Essential Situation 1.9 if and only if the morphism $\xi_{\mathscr{E}\left[p^{m}\right]}: \mathscr{A} \rightarrow \mathscr{B} \mathscr{T}_{m} \otimes_{\mathbb{F}_{p}} k$ defined by $\mathscr{E}\left[p^{m}\right]$ is smooth and contains the image of $\xi$. Note that in this case, the level $m$ stratum $\mathfrak{s}_{D}(m)=\mathscr{A}_{D}\left(\mathscr{E}\left[p^{m}\right]\right)$ is smooth over $\mathscr{G}_{\xi}$ and hence by Lemma 2.1 over $k$. This was claimed in the introduction.

$4^{\mathrm{e}}$ SÉRIE - TOME $43-2010-\mathrm{N}^{\mathrm{o}} 6$ 
To reduce to the Essential Situation 1.9, by Lemma 2.2, we can assume that $k$ is algebraically closed, and it suffices to prove the following proposition.

Proposition 2.3. - There exists a smooth $k$-scheme $\mathscr{A}$ of finite type which is equidimensional of dimension $c d$ and a p-divisible group $\mathscr{E}$ of codimension $c$ and dimension $d$ over $\mathscr{A}$ such that for each $m \in \mathbb{N}^{*}$ the morphism $\xi_{\mathscr{E}\left[p^{m}\right]}: \mathscr{A} \rightarrow \mathscr{B} \mathscr{T}_{m} \otimes_{\mathbb{F}_{p}} k$ defined by $\mathscr{E}\left[p^{m}\right]$ is smooth and surjective.

Proof. - For $\mathscr{A}$, we will take the special fibre of a good integral model of a Shimura variety $\operatorname{Sh}(\mathscr{G}, \mathscr{X})$ associated to a certain PEL-datum as follows. If $c=d=1$, we can take $\operatorname{Sh}(\mathscr{G}, \mathscr{X})$ to be the elliptic modular curve. Thus we can assume that $r:=c+d \geq 3$; in this case the PEL-datum will be unitary.

Let $\mathbb{K}$ be a quadratic imaginary extension of $\mathbb{Q}$ in which $p$ splits. Let $O_{\mathbb{K}}$ be the ring of integers of $\mathbb{K}$. Let ${ }^{*}$ be the nontrivial automorphism of $\mathbb{K}$. Let $\mathbb{V}$ be a $\mathbb{Q}$-vector space of dimension $2 r$. We fix a monomorphism $\mathbb{K} \hookrightarrow \operatorname{End}(\mathbb{V})$ of $\mathbb{Q}$-algebras. Via this monomorphism, we can view $\mathbb{V}$ naturally as a $\mathbb{K}$-vector space of dimension $r$ and we can view $\operatorname{Res}_{\mathbb{K} / \mathbb{Q}} \mathbb{G}_{m, \mathbb{K}}$ as a torus of $\mathbf{G} \mathbf{L}_{\mathbb{V}}$. Whenever we write $\mathbf{S} \mathbf{L}_{\mathbb{V}}$ or $\mathbf{G} \mathbf{L}_{\mathbb{V}}$, we consider $\mathbb{V}$ only as a $\mathbb{Q}$-vector space.

Let $\mathscr{G}^{\text {der }}$ be the simply connected semisimple group over $\mathbb{Q}$ whose $\mathbb{Q}$-valued points are those $\mathbb{K}$-valued points of $\mathbf{S L}_{\mathbb{V}}$ that leave invariant the hermitian form $-z_{1} z_{1}^{*}-\cdots-z_{c} z_{c}^{*}+z_{c+1} z_{c+1}^{*}+\cdots z_{r} z_{r}^{*}$ on $\mathbb{V}$. The group $\mathscr{G}_{\mathbb{R}}^{\text {der }}$ is isomorphic to $\mathbf{S U}(c, d)$. Hilbert's Theorem 90 implies that there exists a unique (up to non-zero scalar multiplication) symplectic form $\langle\rangle:, \mathbb{V} \times \mathbb{V} \rightarrow \mathbb{Q}$ fixed by $\mathscr{G}^{\text {der }}$. We have $\left\langle b v, v^{\prime}\right\rangle=\left\langle v, b^{*} v^{\prime}\right\rangle$ for all $b \in \mathbb{K}$ and $v, v^{\prime} \in \mathbb{V}$.

Let $\mathscr{G}$ be the subgroup of $\operatorname{GSp}(\mathbb{V},<,>)$ generated by $\mathscr{G}$ der and by the torus $\operatorname{Res}_{\mathbb{K} / \mathbb{Q}} \mathbb{G}_{m, \mathbb{K}}$. Our notations match, i.e. $\mathscr{G}^{\text {der }}$ is the derived group of $\mathscr{G}$. It is easy to see that there exists a $\mathscr{G}(\mathbb{R})$-conjugacy class $\mathscr{X}$ of homomorphisms $h: \operatorname{Res}_{\mathbb{C} / \mathbb{R}} \mathbb{G}_{m, \mathbb{C}} \rightarrow \mathscr{G}_{\mathbb{R}}$ such that every $h \in \mathscr{X}$ defines a Hodge $\mathbb{Q}$-structure on $\mathbb{V}$ of type $\{(-1,0),(0,-1)\}$ (with the sign convention of [3]) and such that

$$
\mathbb{V}_{\mathbb{R}} \times \mathbb{V}_{\mathbb{R}} \rightarrow \mathbb{R}, \quad\left(v, v^{\prime}\right) \mapsto\left\langle v, h(\sqrt{-1}) v^{\prime}\right\rangle
$$

is symmetric and either positive or negative definite (for instance, see [3], proof of Proposition 2.3.10 or [12], Lemma 4.3). Then $(\mathscr{G}, \mathscr{X})$ is a Shimura pair given by the PEL-datum $\left(\mathbb{K},{ }^{*}, \mathbb{V},\langle\rangle,, \mathscr{X}\right.$ ). Its reflex field $E$ is either equal to $\mathbb{Q}$ (if $c=d$ ) or isomorphic to $\mathbb{K}$ (if $c \neq d$ ). In both cases, for each prime $v$ of $E$ that divides $p$ the completion $E_{v}$ of $E$ with respect to $v$ is $\mathbb{Q}_{p}$.

As $p$ splits in $\mathbb{K}$, the reductive group $\mathscr{G}_{\mathbb{Q}_{p}}$ is split. This implies that there exists an $O_{\mathbb{K}}$-invariant $\mathbb{Z}_{p}$-lattice $\Gamma$ of $\mathbb{V} \otimes_{\mathbb{Q}} \mathbb{Q}_{p}$ such that the alternating form on $\Gamma$ induced by $\langle$,$\rangle is a perfect$ $\mathbb{Z}_{p}$-form. Denote by $\mathbb{A}_{f}^{(p)}$ the ring of finite adèles of $\mathbb{Q}$ with trivial $p$-th component and fix an open compact subgroup $C^{(p)} \subset \mathscr{G}\left(\mathbb{A}_{f}^{(p)}\right)$. Let $\mathcal{M}$ be the moduli space over $O_{E_{v}}=\mathbb{Z}_{p}$ of abelian schemes associated to the data $\left(\mathbb{K},{ }^{*}, \mathbb{V},\langle\rangle,, O_{\mathbb{K}}, \Gamma, C^{(p)}\right)$, cf. [12], Section 5.

We set $\mathscr{A}:=\mathcal{M} \otimes_{\mathbb{Z}_{p}} k$. For $C^{(p)}$ small enough, $\mathscr{A}$ is quasi-projective, smooth, and equidimensional of dimension $c d$ over $k$. As the adjoint group of $\mathscr{G}$ is simple, the Hasse principle holds for $\mathscr{G}$ (cf. [12], top of p. 394) and this implies that $\mathcal{M}$ is an integral model over $\mathbb{Z}_{p}$ of $\operatorname{Sh}(\mathscr{G}, \mathscr{X})$ alone (cf. [12], Section 7). 
Let $\mathscr{Z}$ be the $p$-divisible group of the universal abelian scheme over $\mathscr{A}$. The height of $\mathscr{Z}$ is $2 r$ and its dimension is $r$. It is endowed with an action of $O_{\mathbb{K}} \otimes_{\mathbb{Z}} \mathbb{Z}_{p}=\mathbb{Z}_{p} \times \mathbb{Z}_{p}$ and with a principal quasi-polarization $\Lambda_{\mathscr{Z}}$. The action of $\mathbb{Z}_{p} \times \mathbb{Z}_{p}$ defines a decomposition $\mathscr{Z}=\mathscr{E} \times \mathscr{E}^{\prime}$, where $\mathscr{E}$ is a $p$-divisible group over $\mathscr{A}$ of codimension $c$ and dimension $d$ and where $\mathscr{E} \prime$ is via $\Lambda_{\mathscr{Z}}$ isomorphic to the Cartier dual $\mathscr{E}^{\vee}$ of $\mathscr{E}$. In particular $\mathscr{Z} \cong \mathscr{E} \times \mathscr{E}^{\vee}$, endowed with its natural $\mathbb{Z}_{p} \times \mathbb{Z}_{p}$-action and with its natural principal quasi-polarization.

Due to the moduli interpretation of $\mathscr{M}$, we easily get that:

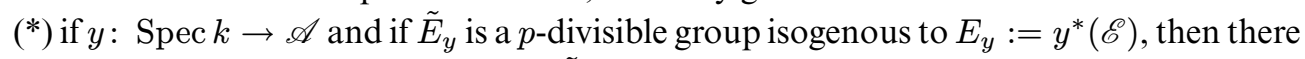
exists a point $\tilde{y}: \operatorname{Spec} k \rightarrow \mathscr{A}$ such that $\tilde{E}_{y}$ is isomorphic to $E_{\tilde{y}}:=\tilde{y}^{*}(\mathscr{E})$.

We claim that the morphism $\xi_{\mathscr{E}\left[p^{m}\right]}: \mathscr{A} \rightarrow \mathscr{B} \mathscr{T}_{m} \otimes_{\mathbb{F}_{p}} k$ is smooth and surjective. To check this, let $R$ be a local, artinian $k$-algebra and let $I \subset R$ be an ideal with $I^{2}=0$. We define $R_{0}:=R / I$. Assume that we are given a commutative diagram

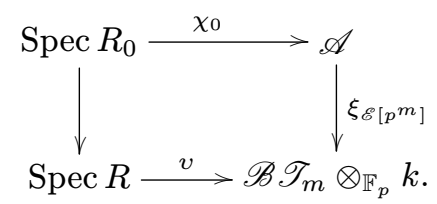

By a theorem of Grothendieck (see [9], Théorème 4.4) there exists a $p$-divisible group $E$ over $R$ which lifts $\chi_{0}^{*}(\mathscr{E})$ and such that $E\left[p^{m}\right]$ is the $\mathrm{BT}_{m}$ corresponding to $v$. We endow $E \times E^{\vee}$ with the natural $\mathbb{Z}_{p} \times \mathbb{Z}_{p}$-action and with its natural principal quasi-polarization. As explained above, we have $\left(E \times E^{\vee}\right) \times_{R} R_{0} \cong \chi_{0}^{*}(\mathscr{Z})$. From this and the Serre-Tate deformation theory, we get that there exists an abelian scheme over $R$ whose $p$-divisible group is $E \times E^{\vee}$ and such that its reduction modulo $I$ is given by $\chi_{0}$, and this, due to the moduli interpretation of $\mathscr{M}$, defines a morphism $\chi: \operatorname{Spec} R \rightarrow \mathscr{A}$ that lifts $\chi_{0}$. Thus $\xi_{\mathscr{E}\left[p^{m}\right]}$ is smooth.

By the generalization of the integral Manin principle to certain Shimura varieties of PEL-type proved in [27], Subsection 5.4 (in particular Example 5.4.3 (b)), $\xi_{\mathscr{E}\left[p^{m}\right]}$ is also surjective. For the reader's convenience and as our present context is much simpler than the general situation considered in [27], we give a direct proof of the surjectivity of $\xi_{\mathscr{E}\left[p^{m}\right]}$.

Let $K$ be an algebraically closed extension of $k$ and let $\tilde{\xi}$ : Spec $K \rightarrow \mathscr{B} \mathscr{T}_{m} \otimes_{\mathbb{F}_{p}} k$ be a $K$-valued point corresponding to a $\mathrm{BT}_{m} \tilde{D}_{m}$ over $K$. Let $\tilde{D}$ be a $p$-divisible group over $K$ such that $\tilde{D}\left[p^{m}\right]=\tilde{D}_{m}$. To show that $\xi_{\mathscr{E}\left[p^{m}\right]}$ is surjective it suffices to prove that there exists a point $y_{3}:$ Spec $K \rightarrow \mathscr{A}$ such that the $p$-divisible groups $y_{3}^{*}(\mathscr{E})$ and $\tilde{D}$ are isomorphic (thus the fibre product of $\tilde{\xi}$ and $\xi_{\mathscr{E}\left[p^{m}\right]}$ is non-empty). We check this in four steps as follows.

(i) Let $\mathscr{T}_{0}$ be a maximal torus of $\mathscr{G}^{\text {der }}$ such that $\mathscr{T}_{0, \mathbb{R}}$ is compact and $\mathscr{T}_{0, \mathbb{Q}_{p}}$ has $\mathbb{Q}_{p}$-rank 0 , cf. [8], Lemma 5.5.3. Let $\mathscr{T}$ be the unique maximal torus of $\mathscr{G}$ that contains $\mathscr{T}_{0}$ (it is generated by $\mathscr{T}_{0}$ and by the center of $\mathscr{G}$ ). Let $h_{0} \in \mathscr{X}$ be such that it factors through $\mathscr{T}_{0}$ (it exists as all maximal compact tori of $\mathscr{G}_{\mathbb{R}}^{\text {der }}$ are $\mathscr{G}^{\mathrm{der}}(\mathbb{R})$-conjugate and as the centralizer of each $h \in \mathscr{X}$ in $\mathscr{G}_{\mathbb{R}}^{\text {der }}$ is a maximal compact, connected subgroup of $\left.\mathscr{G}_{\mathbb{R}}^{\text {der }}\right)$. We have an injective map $\left(\mathscr{T},\left\{h_{0}\right\}\right) \hookrightarrow(\mathscr{G}, \mathscr{X})$ of Shimura pairs. Each point of $\mathscr{M}_{E_{v}}$ which is in the image of the natural functorial morphism $\operatorname{Sh}\left(\mathscr{T},\left\{h_{0}\right\}\right)_{E_{v}} \rightarrow \mathscr{M}_{E_{v}}$ specializes to a point $y_{0}: \operatorname{Spec} k \rightarrow \mathscr{A}$ such that $y_{0}^{*}(\mathscr{E})$ is isoclinic (due to the fact that $\mathscr{T}_{0, \mathbb{Q}_{p}}$ has $\mathbb{Q}_{p}$-rank 0). Such a specialization makes sense as abelian varieties with complex multiplication over number fields have potentially good reduction everywhere. 
(ii) Based on (*) (applied over $K$ ) and (i) we get that there exists a point $y_{1}: \operatorname{Spec} K \rightarrow \mathscr{A}$ such that $y_{1}^{*}(\mathscr{E})$ is isoclinic and its $a$-number is 1 .

(iii) Based on (ii) and Grothendieck's specialization conjecture for $p$-divisible groups over $K$ of $a$-number 1 (proved in [23], Sections 6, 7, and 24; see also [19], Theorem 6.2), there exists a point $y_{2}:$ Spec $K \rightarrow \mathscr{A}$ such that the Newton polygons of $y_{2}^{*}(\mathscr{E})$ and $\tilde{D}$ coincide. Here we are using the fact that $\xi_{\mathscr{E}\left[p^{m}\right]}$ is smooth.

(iv) Based on (iii) and (*) there exists a point $y_{3}: \operatorname{Spec} K \rightarrow \mathscr{A}$ such that the $p$-divisible groups $y_{3}^{*}(\mathscr{E})$ and $\tilde{D}$ are isomorphic.

\section{Group actions}

From now on, we will be in the Essential Situation 1.9. Thus $k$ is algebraically closed and $D$ is a $p$-divisible group over $k$ of codimension $c$ and dimension $d$. The height of $D$ is $r:=c+d$. In this section, we recall from [25] the definition of an action $\mathbb{T}_{m}: \mathscr{H}_{m} \times \mathscr{D}_{m} \rightarrow \mathscr{D}_{m}$ of a linear algebraic group $\mathscr{H}_{m}$ over $k$ on a $k$-scheme $\mathscr{D}_{m}$ whose orbits parameterize isomorphism classes of $\mathrm{BT}_{m}$ 's over $k$.

For a commutative $\mathbb{F}_{p}$-algebra $R$, let $W_{m}(R)$ be the ring of Witt vectors of length $m$ with coefficients in $R$, let $W(R)$ be the ring of Witt vectors with coefficients in $R$, and let $\sigma_{R}$ be the Frobenius endomorphism of either $W_{m}(R)$ or $W(R)$ induced by the Frobenius endomorphism $r \mapsto r^{p}$ of $R$. We set $\sigma:=\sigma_{k}$. Let $B(k)$ be the field of fractions of $W(k)$.

Let $(M, \phi)$ be the contravariant Dieudonné module of $D$. Thus $M$ is a free $W(k)$-module of rank $r$ and $\phi: M \rightarrow M$ is a $\sigma$-linear endomorphism such that we have $p M \subseteq \phi(M)$. Let $\vartheta:=p \phi^{-1}: M \rightarrow M$ be the Verschiebung map of $(M, \phi)$. Let $M=F^{1} \oplus F^{0}$ be a direct sum decomposition such that $\bar{F}^{1}:=F^{1} / p F^{1}$ is the kernel of the reduction modulo $p$ of $\phi$. Let $\bar{F}^{0}:=F^{0} / p F^{0}$. The ranks of $F^{1}$ and $F^{0}$ are $d$ and $c$ (respectively). We have $\phi\left(\frac{1}{p} F^{1} \oplus F^{0}\right)=M$. The decomposition $M=F^{1} \oplus F^{0}$ gives birth naturally to a direct sum decomposition of $W(k)$-modules

$$
\operatorname{End}(M)=\operatorname{Hom}\left(F^{0}, F^{1}\right) \oplus \operatorname{End}\left(F^{1}\right) \oplus \operatorname{End}\left(F^{0}\right) \oplus \operatorname{Hom}\left(F^{1}, F^{0}\right) .
$$

The association $e \rightarrow \phi(e):=\phi \circ e \circ \phi^{-1}$ defines a $\sigma$-linear automorphism $\phi: \operatorname{End}(M)\left[\frac{1}{p}\right] \stackrel{\sim}{\rightarrow}$ $\operatorname{End}(M)\left[\frac{1}{p}\right]$ of $B(k)$-algebras.

Let $\mathscr{W}_{+}$be the maximal subgroup scheme of $\mathbf{G L}_{M}$ that fixes both $F^{1}$ and $M / F^{1}$; it is a closed subgroup scheme of $\mathbf{G L}_{M}$ whose Lie algebra is the direct summand $\operatorname{Hom}\left(F^{0}, F^{1}\right)$ of $\operatorname{End}(M)$ and whose relative dimension is $c d$. Let $\mathscr{W}_{0}:=\mathbf{G L}_{F^{1}} \times_{W(k)} \mathbf{G L}_{F^{0}}$; it is a closed subgroup scheme of $\mathbf{G L}_{M}$ whose Lie algebra is the direct summand $\operatorname{End}\left(F^{1}\right) \oplus \operatorname{End}\left(F^{0}\right)$ of $\operatorname{End}(M)$ and whose relative dimension is $d^{2}+c^{2}$. The maximal parabolic subgroup scheme $\mathscr{W}_{+0}$ of $\mathbf{G L}_{M}$ that normalizes $F^{1}$ is the semidirect product of $\mathscr{W}_{+}$and $\mathscr{W}_{0}$. Let $\mathscr{W}_{-}$ be the maximal subgroup scheme of $\mathbf{G L}_{M}$ that fixes $F^{0}$ and $M / F^{0}$; it is a closed subgroup scheme of $\mathbf{G L}_{M}$ whose Lie algebra is the direct summand $\operatorname{Hom}\left(F^{1}, F^{0}\right)$ of $\operatorname{End}(M)$ and whose relative dimension is $c d$. The maximal parabolic subgroup scheme $\mathscr{W}_{0-}$ of $\mathbf{G L}_{M}$ that normalizes $F^{0}$ is the semidirect product of $\mathscr{W}_{-}$and $\mathscr{W}_{0}$. If $R$ is a commutative $W(k)$-algebra, then we have:

$$
\begin{aligned}
& \mathscr{W}_{+}(R)=1_{M \otimes_{W(k)} R}+\operatorname{Hom}\left(F^{0}, F^{1}\right) \otimes_{W(k)} R \\
& \mathscr{W}_{-}(R)=1_{M \otimes_{W(k)} R}+\operatorname{Hom}\left(F^{1}, F^{0}\right) \otimes_{W(k)} R .
\end{aligned}
$$


These identities imply that the group schemes $\mathscr{W}_{+}$and $\mathscr{W}_{-}$are isomorphic to $\mathbb{G}_{a}^{c d}$ over Spec $W(k)$; in particular, they are smooth and commutative. Let

$$
\mathscr{H}:=\mathscr{W}_{+} \times_{W(k)} \mathscr{W}_{0} \times_{W(k)} \mathscr{W}_{-}
$$

it is a smooth, affine scheme over Spec $W(k)$ of relative dimension $c d+d^{2}+c^{2}+c d=r^{2}$. We consider the natural product morphism $\mathscr{P}_{0}: \mathscr{H} \rightarrow \mathbf{G L}_{M}$ and the following morphism $\mathscr{P}_{-}:=1_{\mathscr{W}_{+}} \times 1_{\mathscr{W}_{0}} \times p 1_{\mathscr{W}_{-}}: \mathscr{H} \rightarrow \mathscr{H}$. Let

$$
\mathscr{P}_{0-}:=\mathscr{P}_{0} \circ \mathscr{P}_{-}: \mathscr{H} \rightarrow \mathbf{G L}_{M}
$$

it is a morphism of Spec $W(k)$-schemes whose generic fibre is an open embedding of Spec $B(k)$-schemes.

Let $\tilde{\mathscr{H}}$ be the dilatation of $\mathrm{GL}_{M}$ centered on $\mathscr{W}_{+0, k}$ (see [1], Chapter 3, Section 3.2 for dilatations). We recall that if $\mathbf{G} \mathbf{L}_{M}=\operatorname{Spec} R_{M}$ and if $I_{+0 k}$ is the ideal of $R_{M}$ that defines $\mathscr{W}_{+0, k}$, then as a scheme $\tilde{\mathscr{H}}$ is the spectrum of the $R_{M}$-subalgebra $R_{\tilde{\mathscr{H}}}$ of $R_{M}\left[\frac{1}{p}\right]$ generated by all elements $\frac{*}{p}$ with $* \in I_{+0 k}$. It is well known that $\tilde{\mathscr{H}}$ is a smooth, affine group scheme over Spec $W(k)$ which is uniquely determined by the following two additional properties (they follow directly from the definition of $R_{\tilde{\mathscr{C}}}$; see Propositions 1, 2, and 3 of loc. cit.):

(i) There exists a homomorphism $\tilde{\mathscr{P}}_{0-}: \tilde{\mathscr{H}} \rightarrow \mathbf{G L}_{M}$ whose generic fibre is an isomorphism of Spec $B(k)$-schemes.

(ii) A morphism $f: Y \rightarrow \mathbf{G L}_{M}$ of flat Spec $W(k)$-schemes factors (uniquely) through $\tilde{\mathscr{P}}_{0-}$ if and only if the morphism $f_{k}: Y_{k} \rightarrow \mathbf{G L}_{M / p M}$ factors through $\mathscr{W}_{+0, k}$.

The group $\tilde{\mathscr{H}}(W(k))$ is the parahoric subgroup of $\mathbf{G L}_{M}(W(k))$ that normalizes the sublattice $F^{1} \oplus p F^{0}=F^{1}+p M$ of $M$.

The morphism $\mathscr{P}_{0-}$ factors naturally as a morphism $\mathscr{P}: \mathscr{H} \rightarrow \tilde{\mathscr{H}}$ (cf. (ii)) whose $p$-adic completion is an isomorphism (cf. [25], Subsubsection 2.1.1). Therefore we have a natural identification $\mathscr{H}_{W_{m}(k)}=\tilde{\mathscr{H}}_{W_{m}(k)}$ that provides $\mathscr{H}_{W_{m}(k)}$ with a group scheme structure over $\operatorname{Spec} W_{m}(k)$ which does not depend on the decomposition $\mathscr{H}=\mathscr{W}_{+} \times_{W(k)} \mathscr{W}_{0} \times_{W(k)} \mathscr{W}_{-}$ produced by the choice of the direct sum decomposition $M=F^{1} \oplus F^{0}$.

For $g \in \mathbf{G L}_{M}(W(k))$ and $h=\left(h_{1}, h_{2}, h_{3}\right) \in \mathscr{H}(W(k))$, let $g[m] \in \mathbf{G L}_{M}\left(W_{m}(k)\right)$ and $h[m]=\left(h_{1}[m], h_{2}[m], h_{3}[m]\right) \in \mathscr{H}\left(W_{m}(k)\right)$ be the reductions modulo $p^{m}$ of $g$ and $h$ (respectively). Thus $1_{M / p^{m} M}=1_{M}[m]$. Let $\phi_{m}, \vartheta_{m}: M / p^{m} M \rightarrow M / p^{m} M$ be the reductions modulo $p^{m}$ of $\phi, \vartheta: M \rightarrow M$.

Let $\sigma_{\phi}: M \stackrel{\sim}{\rightarrow} M$ be the $\sigma$-linear automorphism which takes $x \in F^{1}$ to $\frac{1}{p} \phi(x)$ and takes $x \in F^{0}$ to $\phi(x)$. Let $\sigma_{\phi}$ act on the sets underlying the groups $\mathbf{G L}_{M}(W(k))$ and $\mathbf{G L}_{M}\left(W_{m}(k)\right)$ in the natural way: if $g \in \mathbf{G L}_{M}(W(k))$, then $\sigma_{\phi}(g):=\sigma_{\phi} g \sigma_{\phi}^{-1}$ and $\sigma_{\phi}(g[m]):=\left(\sigma_{\phi} g \sigma_{\phi}^{-1}\right)[m]$. For $g \in \mathscr{W}_{+}(W(k))$ (resp. $g \in \mathscr{W}_{0}(W(k))$ or $\left.g \in \mathscr{W}_{-}(W(k))\right)$ we have $\phi(g)=\sigma_{\phi}\left(g^{p}\right)$ (resp. we have $\phi(g)=\sigma_{\phi}(g)$ or $\left.\phi\left(g^{p}\right)=\sigma_{\phi}(g)\right)$.

Let Aff $k$ be the category of affine schemes over $k$. Let Set and Group be the categories of abstract sets and groups (respectively). Let $\Delta$ be a smooth, affine (resp. a smooth, affine group) scheme of finite type over $\operatorname{Spec} W(k)$. Let $\mathbb{W}_{m}(\Delta):$ Aff $_{k} \rightarrow$ Set (resp. $\mathbb{W}_{m}(\Delta):$ Aff $_{k} \rightarrow$ Group) be the contravariant functor that associates to an affine $k$-scheme Spec $R$ the set (resp. the group) $\Delta\left(W_{m}(R)\right)$. This functor is representable by an affine, smooth (resp. affine, smooth group) scheme over $k$ of finite type to be denoted also by $\mathbb{W}_{m}(\triangle)$ (see [25], Subsection 2.1.4 for these facts due to Greenberg). 
Let $\mathscr{H}_{m}:=\mathbb{W}_{m}(\mathscr{H})$ and $\mathscr{D}_{m}:=\mathbb{W}_{m}\left(\mathbf{G L}_{M}\right)$. As $\mathscr{P}_{W_{m}(k)}: \mathscr{H}_{W_{m}(k)} \rightarrow \tilde{\mathscr{H}}_{W_{m}(k)}$ is an isomorphism of Spec $W_{m}(k)$-schemes, we will identify naturally

$$
\mathscr{H}(W(k))=\tilde{\mathscr{H}}(W(k)) \text { and } \mathscr{H}_{m}=\mathbb{W}_{m}(\mathscr{H})=\mathbb{W}_{m}(\tilde{\mathscr{H}}) .
$$

Thus in what follows we will view $\mathscr{H}(W(k))$ as a subgroup of $\mathbf{G L}_{M}(W(k))$ and $\mathscr{H}_{m}$ as a connected, smooth, affine group over $k$ of dimension $m r^{2}$ (cf. [25], Subsection 2.1.4 applied to $\tilde{\mathscr{H}}$ ). Similarly, we will view $\mathscr{D}_{m}$ as a connected, smooth, affine variety over $k$ of dimension $m r^{2}$. Let

$$
\mathbb{T}_{m}: \mathscr{H}_{m} \times_{k} \mathscr{D}_{m} \rightarrow \mathscr{D}_{m}
$$

be the action defined on $k$-valued points as follows. If $h=\left(h_{1}, h_{2}, h_{3}\right) \in \mathscr{H}(W(k))$ and $g \in \mathbf{G L}_{M}(W(k))$, then the product of $h[m]=\left(h_{1}[m], h_{2}[m], h_{3}[m]\right) \in \mathscr{H}_{m}(k)=\mathscr{H}\left(W_{m}(k)\right)$ and $g[m] \in \mathscr{D}_{m}(k)=\mathbf{G L}_{M}\left(W_{m}(k)\right)$ is the element

$$
\begin{gathered}
\mathbb{T}_{m}(h[m], g[m]):=\left(h_{1} h_{2} h_{3}^{p} g \phi\left(h_{1} h_{2} h_{3}^{p}\right)^{-1}\right)[m] \\
=\left(h_{1} h_{2} h_{3}^{p} g \phi\left(h_{3}^{p}\right)^{-1} \phi\left(h_{2}\right)^{-1} \phi\left(h_{1}\right)^{-1}\right)[m]=\left(h_{1} h_{2} h_{3}^{p} g \sigma_{\phi}\left(h_{3}\right)^{-1} \sigma_{\phi}\left(h_{2}\right)^{-1} \sigma_{\phi}\left(h_{1}^{p}\right)^{-1}\right)[m] \\
=h_{1}[m] h_{2}[m] h_{3}[m]^{p} g[m] \sigma_{\phi}\left(h_{3}[m]\right)^{-1} \sigma_{\phi}\left(h_{2}[m]\right)^{-1} \sigma_{\phi}\left(h_{1}[m]^{p}\right)^{-1} \in \mathscr{D}_{m}(k) .
\end{gathered}
$$

The formula $\mathbb{T}_{m}(h[m], g[m])=\left(h_{1} h_{2} h_{3}^{p} g \phi\left(h_{1} h_{2} h_{3}^{p}\right)^{-1}\right)[m]$ shows that the action $\mathbb{T}_{m}$ is intrinsically associated to $D$, i.e. it does not depend on the choice of the direct sum decomposition $M=F^{1} \oplus F^{0}$.

Let $\mathscr{O}_{m}$ be the orbit of $1_{M}[m] \in \mathscr{D}_{m}(k)$ under the action $\mathbb{T}_{m}$. Let $\overline{\mathscr{O}}_{m}$ be the schemetheoretic closure of $\mathscr{O}_{m}$ in $\mathscr{D}_{m}$; it is an affine, integral scheme over $k$. The orbit $\mathscr{O}_{m}$ is a connected, smooth, open subscheme of $\overline{\mathscr{O}}_{m}$ and thus it is also a quasi-affine scheme over $k$. Let $\mathscr{S}_{m}$ be the subgroup scheme of $\mathscr{H}_{m}$ which is the stabilizer of $1_{M}[m]$ under the action $\mathbb{T}_{m}$. Let $\mathscr{C}_{m}$ be the reduced group of $\mathscr{S}_{m}$. Let $\mathscr{C}_{m}^{0}$ be the identity component of $\mathscr{C}_{m}$. The connected, smooth group $\mathscr{C}_{m}^{0}$ over $k$ is unipotent, i.e. it has no torus of positive dimension (see [25], Theorem 2.4 (a); see also Subsection 4.3).

\section{Combinatorics of the action $\mathbb{T}_{1}$}

In this section, we present basic combinatorial properties of the action $\mathbb{T}_{1}$ which will be used in Section 5 to show that if the condition (C) holds for $D[p]$, then the orbit $\mathscr{O}_{m}$ of $\mathbb{T}_{m}$ is affine. For the remaining part of this section, we let $m=1$ and we use the notations of Section 3.

\subsection{Nilpotent subalgebras of $\operatorname{End}(M / p M)$}

In [13] (see also [20], Subsection 2.3 and Lemma 2.4) it is shown that there exist a $k$-basis $\left\{\bar{e}_{1}, \ldots, \bar{e}_{r}\right\}$ for $M / p M$ and a permutation $\pi$ of the set $J:=\{1, \ldots, r\}$ such that for each $i \in J$, the following two properties hold:

(i) $\phi_{1}\left(\bar{e}_{i}\right)=0$ if $i>c$, and $\phi_{1}\left(\bar{e}_{i}\right)=\bar{e}_{\pi(i)}$ if $i \leq c$;

(ii) $\vartheta_{1}\left(\bar{e}_{\pi(i)}\right)=0$ if $i \leq c$, and $\vartheta_{1}\left(\bar{e}_{\pi(i)}\right)=\bar{e}_{i}$ if $i>c$. 
The permutation $\pi$ is not uniquely determined by the isomorphism class of $D[p]$. For instance, we can always replace $\pi$ by $\pi_{0} \pi_{1} \pi \pi_{0}^{-1}$, where $\pi_{0}$ is an arbitrary permutation of the set $J$ that leaves invariant the subset $\{1, \ldots, c\}$ and where $\pi_{1}$ is an arbitrary permutation of the set $J$ that leaves invariant all the subsets $\left\{\pi^{i}(1), \ldots, \pi^{i}(c)\right\}$ with $i \in \mathbb{N}$. More precisely, it is known (e.g., [16], [26], or [17]) that there exists a canonical bijection between isomorphism classes of $\mathrm{BT}_{1}$ 's over $k$ and the quotient set $S_{r} /\left(S_{c} \times S_{d}\right)$.

Let $\left\{e_{1}, \ldots, e_{r}\right\}$ be a $W(k)$-basis for $M$ that lifts the $k$-basis $\left\{\bar{e}_{1}, \ldots, \bar{e}_{r}\right\}$ for $M / p M$ and such that $F^{1}=\oplus_{i=c+1}^{r} W(k) e_{i}$. Let $\left\{e_{i, j} \mid i, j \in J\right\}$ be the $W(k)$-basis for $\operatorname{End}(M)$ such that for each $l \in J$ we have $e_{i, j}\left(e_{l}\right)=\delta_{j, l} e_{i}$. Let $\left\{\bar{e}_{i, j} \mid i, j \in J\right\}$ be the reduction modulo $p$ of $\left\{e_{i, j} \mid i, j \in J\right\}$; it is a $k$-basis for $\operatorname{End}(M / p M)$. Let $\sigma_{\pi}: M \stackrel{\sim}{\rightarrow} M$ be the $\sigma$-linear automorphism that maps $e_{i}$ to $e_{\pi(i)}$ for all $i \in J$. Let $g_{\pi}:=\sigma_{\pi} \sigma_{\phi}^{-1} \in \mathbf{G L}_{M}(W(k))$. Due to the properties (i) and (ii), the reduction modulo $p$ of $\left(M, g_{\pi} \phi, \vartheta g_{\pi}^{-1}\right)$ coincides with $\left(M / p M, \phi_{1}, \vartheta_{1}\right)$. Based on this, we can assume that $g_{\pi}[1]=1_{M}[1]$; thus $\sigma_{\phi}$ and $\sigma_{\pi}$ are congruent modulo $p$. As the action $\mathbb{T}_{1}$ is intrinsically associated to $D$ (i.e., it does not depend on the choice of the direct sum decomposition $M=F^{1} \oplus F^{0}$ ), to study the group $\mathscr{C}_{1}^{0}$ we can assume $F^{0}=\oplus_{i=1}^{c} W(k) e_{i}$. Let

$$
\mathscr{J}_{+}:=\left\{(i, j) \in J^{2} \mid j \leq c<i\right\},
$$

$\mathscr{J}_{0}:=\left\{(i, j) \in J^{2} \mid\right.$ either $i, j>c$ or $\left.i, j \leq c\right\}, \quad$ and $\quad \mathscr{J}_{-}:=\left\{(i, j) \in J^{2} \mid i \leq c<j\right\}$.

The three sets $\left\{\bar{e}_{i, j} \mid(i, j) \in \mathscr{J}_{+}\right\},\left\{\bar{e}_{i, j} \mid(i, j) \in \mathscr{J}_{0}\right\}$, and $\left\{\bar{e}_{i, j} \mid(i, j) \in \mathscr{J}_{-}\right\}$are $k$-bases for $\operatorname{Lie}\left(\mathscr{W}_{+, k}\right)=\operatorname{Hom}\left(\bar{F}^{0}, \bar{F}^{1}\right), \operatorname{Lie}\left(\mathscr{W}_{0, k}\right)=\operatorname{End}\left(\bar{F}^{1}\right) \oplus \operatorname{End}\left(\bar{F}^{0}\right)$, and $\operatorname{Lie}\left(\mathscr{W}_{-, k}\right)=$ $\operatorname{Hom}\left(\bar{F}^{1}, \bar{F}^{0}\right)$ (respectively).

By the $\pi$-order of the pair $(i, j) \in \mathscr{J}_{-}$, we mean the smallest positive integer $\nu(i, j)$ such that we have

$$
\left(\pi^{\nu(i, j)}(i), \pi^{\nu(i, j)}(j)\right) \in \mathscr{J}_{+} \cup \mathscr{J}_{-} .
$$

We define the following five sets:

$$
\begin{gathered}
\mathscr{J}_{-, 1}:=\left\{(i, j) \in \mathscr{J}_{-} \mid\left(\pi^{\nu(i, j)}(i), \pi^{\nu(i, j)}(j)\right) \in \mathscr{J}_{+}\right\} \quad \text { and } \mathscr{J}_{-, 2}:=\mathscr{J}_{-} \backslash \mathscr{J}_{-, 1} \\
\mathscr{J}_{+, 1}:=\left\{\left(\pi^{\nu(i, j)}(i), \pi^{\nu(i, j)}(j)\right) \mid(i, j) \in \mathscr{J}_{-, 1}\right\} \text { and } \mathscr{J}_{+, 2}:=\mathscr{J}_{+} \backslash \mathscr{J}_{+, 1} \\
\mathscr{J}_{0,0}:=\left\{\left(\pi^{s}(i), \pi^{s}(j) \mid(i, j) \in \mathscr{J}_{-, 1} \text { and } s \in\{1, \ldots, \nu(i, j)-1\}\right\} .\right.
\end{gathered}
$$

We remark that the set $\mathscr{J}_{+} \cup \mathscr{J}_{0,0} \cup \mathscr{J}_{-}$contains no pair of the form $(i, i)$. The number of elements of the set $\mathscr{J}_{0,0}$ is

$$
\left|\mathscr{J}_{0,0}\right|:=\sum_{(i, j) \in \mathscr{J}_{-, 1}}(\nu(i, j)-1) .
$$

For $(i, j) \in \mathscr{J}_{-, 1}$ and $s \in\{0,1, \ldots, \nu(i, j)\}$ we define the $\pi$-level of $\left(\pi^{s}(i), \pi^{s}(j)\right)$ to be the number $\eta\left(\pi^{s}(i), \pi^{s}(j)\right):=s$ and we define the $\pi$-order of $\left(\pi^{s}(i), \pi^{s}(j)\right)$ to be the number $\nu\left(\pi^{s}(i), \pi^{s}(j)\right):=\nu(i, j)-s$. Thus the $\pi$-order $\nu(i, j)$ and the $\pi$-level $\eta(i, j)$ are well defined for all pairs $(i, j) \in \mathscr{J}_{+, 1} \cup \mathscr{J}_{0,0} \cup \mathscr{J}_{-, 1}$.

Lemma 4.1. - Let $\mathfrak{n}_{\star}$ be the $k$-vector space generated by those $\bar{e}_{(i, j)}$ 's with $(i, j) \in \mathscr{J}_{\star}$ (thus $\mathfrak{n}_{+, 1}:=\oplus_{(i, j) \in \mathscr{J}_{+, 1}} k \bar{e}_{i, j}, \mathfrak{n}_{0,0}:=\oplus_{(i, j) \in \mathscr{J}_{0,0}} k \bar{e}_{i, j}$, etc.). Then the following four properties hold:

$4^{\text {e }}$ SÉRIE - TOME $43-2010-\mathrm{N}^{\mathrm{o}} 6$ 
(a) If the pairs $(i, j)$ and $(j, l)$ belong to $\mathscr{J}_{0,0}$, then we have $(i, l) \in \mathscr{J}_{0,0}$. Similarly, if one of the pairs $(i, j)$ and $(j, l)$ belongs to $\mathscr{J}_{0,0}$ and the other one belongs to $\mathscr{J}_{+, 1}$ (resp. to $\left.\mathscr{J}_{-, 1}\right)$, then we have $(i, l) \in \mathscr{J}_{+, 1}$ (resp. we have $(i, l) \in \mathscr{J}_{-, 1}$ ).

(b) The $k$-vector space $\mathfrak{n}_{0,0}$ is a nilpotent subalgebra of $\operatorname{End}(M / p M)$. More precisely, we have $\mathfrak{n}_{0,0}^{\max (c, d)}=0$.

(c) The $k$-vector spaces $\mathfrak{n}_{+}, \mathfrak{n}_{+, 1}, \mathfrak{n}_{-}$, and $\mathfrak{n}_{-, 1}$ are both left and right $\mathfrak{n}_{0,0}$-modules. Moreover we have $\mathfrak{n}_{0,0}^{d} \mathfrak{n}_{+}=\mathfrak{n}_{+} \mathfrak{n}_{0,0}^{c}=\mathfrak{n}_{0,0}^{c} \mathfrak{n}_{-}=\mathfrak{n}_{-} \mathfrak{n}_{0,0}^{d}=0$.

(d) The k-vector spaces $\mathfrak{n}_{+} \oplus \mathfrak{n}_{0,0}, \mathfrak{n}_{+, 1} \oplus \mathfrak{n}_{0,0}, \mathfrak{n}_{0,0} \oplus \mathfrak{n}_{-}$, and $\mathfrak{n}_{0,0} \oplus \mathfrak{n}_{-, 1}$ are nilpotent subalgebras of $\operatorname{End}(M / p M)$.

Proof. - We prove only the first part of (a) as the second part of (a) is proved similarly. Let $s:=\min \{\eta(i, j), \eta(j, l)\} \in \mathbb{N}^{*}$ and let $t:=\min \{\nu(i, j), \nu(j, l)\} \in \mathbb{N}^{*}$. From the very definition of $s$, we get that one of the two pairs $\left(\pi^{-s}(i), \pi^{-s}(j)\right)$ and $\left(\pi^{-s}(j), \pi^{-s}(l)\right)$ belongs to $\mathscr{J}_{-}$while the other one belongs to $\mathscr{J}_{0}$; thus $\left(\pi^{-s}(i), \pi^{-s}(l)\right) \in \mathscr{J}_{-}$. It is easy to see that for all $u \in\{1, \ldots, s+t-1\}$ we have $\left(\pi^{-s+u}(i), \pi^{-s+u}(l)\right) \in \mathscr{J}_{0}$. From the very definition of $t$, we get that one of the two pairs $\left(\pi^{t}(i), \pi^{t}(j)\right)$ and $\left(\pi^{t}(j), \pi^{t}(l)\right)$ belongs to $\mathscr{J}_{+}$while the other one belongs to $\mathscr{J}_{0}$; thus $\left(\pi^{t}(i), \pi^{t}(l)\right) \in \mathscr{J}_{+}$. From the last three sentences we get that we have $\left(\pi^{-s}(i), \pi^{-s}(l)\right) \in \mathscr{J}_{-, 1}$ and $\nu\left(\pi^{-s}(i), \pi^{-s}(l)\right)=s+t$. As $1 \leq s<s+t$, we have $(i, l) \in \mathscr{J}_{0,0}$ (i.e., the first part of (a) holds) as well as $\eta(i, l)=s$ and $\nu(i, l)=t$.

We check (b). For $\nu \in \mathbb{N}$ let $\mathfrak{n}_{0,0}^{\nu}$ be the $k$-span of those $\bar{e}_{i, j}$ 's for which $(i, j) \in \mathscr{J}_{0,0}$ and $\nu(i, j)=\nu$. As $\mathfrak{n}_{+} \mathfrak{n}_{+}=0$, we have $\mathfrak{n}_{0,0}^{\nu} \mathfrak{n}_{0,0}^{\nu}=0$. From the end of the previous paragraph we get that for $\nu_{1}<\nu_{2}$, we have: $\mathfrak{n}_{0,0}^{\nu_{1}} \mathfrak{n}_{0,0}^{\nu_{2}}+\mathfrak{n}_{0,0}^{\nu_{2}} \mathfrak{n}_{0,0}^{\nu_{1}} \subseteq \mathfrak{n}_{0,0}^{\nu_{1}}$. These imply that $\mathfrak{n}_{0,0}$ is a nilpotent subalgebra of $\operatorname{End}(M / p M)$. Thus $\mathfrak{n}_{0,0}$ is the Lie algebra of the smooth, connected subgroup of $\mathscr{W}_{0, k}=\mathbf{G L}_{\bar{F}^{1}} \times_{k} \mathbf{G L}_{\bar{F}^{0}}$ whose group of valued points in an arbitrary commutative $k$-algebra $R$ is $1_{M \otimes_{W(k)} R}+\mathfrak{n}_{0,0} \otimes_{k} R$. Therefore $\mathfrak{n}_{0,0}$ is $\mathscr{W}_{0}(k)$-conjugate to a Lie subalgebra of $\operatorname{End}\left(\bar{F}^{1}\right) \oplus \operatorname{End}\left(\bar{F}^{0}\right)$ formed by upper triangular nilpotent matrices. This implies that $\mathfrak{n}_{0,0}^{\max (c, d)}=0$. Thus (b) holds.

It is obvious that $\mathfrak{n}_{+}$and $\mathfrak{n}_{-}$are left and right $\mathfrak{n}_{0,0}$-modules. As in the previous paragraph, using the second part of (a) we argue that $\mathfrak{n}_{+, 1}$ and $\mathfrak{n}_{-, 1}$ are left and right $\mathfrak{n}_{0,0}$-modules. The second part of (c) follows from relations of the form $\mathfrak{n}_{0,0}^{d} \mathfrak{n}_{+} \subseteq\left[\mathfrak{n}_{0,0} \cap \operatorname{End}\left(\bar{F}^{1}\right)\right]^{d} \operatorname{Hom}\left(\bar{F}^{0}, \bar{F}^{1}\right)=0$. Thus (c) holds.

Part (d) follows from (b) and (c) and the fact that $\mathfrak{n}_{+}^{2}=\mathfrak{n}_{-}^{2}=0$.

\subsection{Condition (C)}

We define four subsets of $\cup_{s \geq 2} J^{s}$ as follows:

$$
\begin{aligned}
\Gamma & :=\left\{\left(i_{1}, \ldots, i_{s}\right) \mid s \geq 2,\left(i_{\ell}, i_{\ell+1}\right) \in \mathscr{J}_{0,0} \forall \ell \in\{1, \ldots, s-2\},\left(i_{s-1}, i_{s}\right) \in \mathscr{J}_{+, 2}\right\} \\
\Delta & :=\left\{\left(i_{1}, \ldots, i_{s}\right) \mid s \geq 3,\left(i_{1}, \ldots, i_{s-1}\right) \in \Gamma,\left(i_{s-1}, i_{s}\right) \in \mathscr{J}_{0,0}\right\}
\end{aligned}
$$




$$
\begin{aligned}
& \Gamma_{1}:=\left\{\left(i_{1}, \ldots, i_{s}\right) \in \Gamma \mid\left(i_{1}, i_{s}\right) \in \mathscr{J}_{+, 1} \text { and }\left(i_{2}, i_{s}\right) \notin \mathscr{J}_{+, 1}\right\} \\
& \Delta_{1}:=\left\{\left(i_{1}, \ldots, i_{s}\right) \in \Delta \mid\left(i_{1}, i_{s}\right) \in \mathscr{J}_{+, 1} \text { and }\left(i_{2}, i_{s}\right) \notin \mathscr{J}_{+, 1}\right\} .
\end{aligned}
$$

If $\left(i_{1}, \ldots, i_{s}\right) \in \Gamma_{1}$, then $s \geq 3$. For each element $\gamma=\left(i_{1}, \ldots, i_{s}\right) \in \Gamma \cup \Delta$ and for every $t \in \mathbb{N}^{*}$, let $n_{t}(\gamma)$ be the number of elements $\ell \in\{1, \ldots, s-1\}$ such that we have $\left(i_{\ell}, i_{\ell+1}\right) \in \mathscr{J}_{0,0}$ and $\nu\left(i_{\ell}, i_{\ell+1}\right)=t$. We define a number:

$$
\kappa(\gamma):=\sum_{t=1}^{\infty} n_{t}(\gamma) p^{-t} \in(0, \infty) \cap \mathbb{Q}
$$

Definition 4.2. - Let $\kappa(\pi):=\max \left\{\kappa(\gamma) \mid \gamma \in \Gamma_{1} \cup \Delta_{1}\right\}$ with the convention that $\kappa(\pi)=0$ if $\Gamma_{1} \cup \Delta_{1}$ is the empty set. Let $\kappa(D[p])$ be the smallest value $\kappa(\pi)$, where $\pi$ runs through all permutations of the set $\{1, \ldots, r\}$ for which there exists a $k$-basis $\left\{\bar{e}_{1}, \ldots, \bar{e}_{r}\right\}$ for $M / p M$ as in the beginning of Subsection 4.1. We say the condition (C) holds for $D$ (or for any truncation of $D$ ) if either $\kappa(D[p])<1$ or $\kappa\left(D^{\vee}[p]\right)<1$. Here $D^{\vee}$ is the Cartier dual of $D$. We say the condition (C) holds for an $m$-truncated Barsotti-Tate group $B$ over a field $K$ of characteristic $p$, if the condition (C) holds for the extension of $B$ to an algebraic closure of $K$.

Lemma 4.3. - The following four properties hold:

(a) Let $\gamma \in \Gamma$. Then for each positive integer $t$ we have the following inequality $n_{t}(\gamma) \leq 1+\sum_{u=1}^{t-1} n_{u}(\gamma)$. Therefore we have $n_{t}(\gamma) \leq 2^{t-1}$.

(b) If $p \geq 3$ and $\gamma \in \Gamma$, then $\kappa(\gamma)<1$.

(c) If $p \geq 5$ and $\gamma \in \Gamma \cup \Delta$, then $\kappa(\gamma)<1$. Thus if $p \geq 5$, then $\kappa(\pi)<1$ and therefore condition (C) holds for D.

(d) We assume that $p=3$. Then $\kappa(\pi)<\frac{4}{3}$. If moreover $\min \{c, d\} \leq 6$, then condition (C) holds for D.

Proof. - To prove (a) we write $\gamma=\left(i_{1}, \ldots, i_{s}\right)$ and we can assume that $n_{t}(\gamma)>0$; thus we have $s \geq 3$ even if $\gamma \in \Gamma$. We consider the identity

$$
\bar{e}_{\pi^{t}\left(i_{1}\right), \pi^{t}\left(i_{s-1}\right)}=\prod_{\ell=1}^{s-2} \bar{e}_{\pi^{t}\left(i_{\ell}\right), \pi^{t}\left(i_{\ell+1}\right)}
$$

between elements of $\operatorname{End}(M / p M)$. The right-hand side of (1) contains at least $n_{t}(\gamma)$ elements of $\mathfrak{n}_{+}$, it contains $\sum_{u>t} n_{u}(\gamma)$ elements of $\mathfrak{n}_{0,0}$, and it contains $n_{1}(\gamma)+n_{2}(\gamma)+\cdots+n_{t-1}(\gamma)$ elements that belong to $\mathscr{J}_{+}, \mathscr{J}_{0}$, or $\mathscr{J}_{-}$. Let $\bar{\mu}: \mathbb{G}_{m} \rightarrow \mathbf{G L}_{M / p M}$ be the cocharacter that fixes $\bar{F}^{0}$ and that acts via the inverse of the identical character of $\mathbb{G}_{m}$ on $\bar{F}^{1}$. If we have $n_{t}(\gamma)>1+n_{1}(\gamma)+n_{2}(\gamma)+\cdots+n_{t-1}(\gamma)$, then $\mathbb{G}_{m}$ acts via $\bar{\mu}$ on the right-hand side of (1) via at least the second power of the inverse of the identical character of $\mathbb{G}_{m}$; therefore the right-hand side of (1) must be 0 and this contradicts the fact that the left-hand side of (1) is non-zero. Thus we have $n_{t}(\gamma) \leq 1+n_{1}(\gamma)+n_{2}(\gamma)+\cdots+n_{t-1}(\gamma)$. By induction on $t \in \mathbb{N}^{*}$ we get that $n_{t}(\gamma) \leq 2^{t-1}=1+1+2+\cdots+2^{t-2}$. This proves (a).

We prove (b). Due to (a) and the inequality $p \geq 3$ we have

$$
\kappa(\gamma)=\sum_{t=1}^{\infty} n_{t}(\gamma) p^{-t}<\sum_{t=1}^{\infty} 2^{t-1} p^{-t}=\frac{1}{p-2} \leq 1 .
$$

Thus (b) holds. 
Due to (b), to prove (c) we can assume that $\gamma=\left(i_{1}, \ldots, i_{s}\right) \in \Delta$. We have

$$
\kappa(\gamma)=\frac{1}{p^{\nu\left(i_{s-1}, i_{s}\right)}}+\kappa\left(\left(i_{1}, \ldots, i_{s-1}\right)\right) \leq \frac{1}{p}+\kappa\left(\left(i_{1}, \ldots, i_{s-1}\right)\right) .
$$

Due to the last inequality and the fact that $p \geq 5$, from relations (2) and (3) we get that $\kappa(\gamma)<\frac{1}{p-2}+\frac{1}{p}<1$. Thus (c) holds.

The first part of (d) follows from (b) and (3). To check the last part of (d) we can assume that $d \leq 6$ (otherwise we can replace $D$ by $D^{\vee}$ ). From Lemma 4.1 (c), (by taking $t=0$ in Formula (1)) we get that for each element $\gamma=\left(i_{1}, \ldots, i_{s}\right) \in \Gamma$ we have $s \leq d+1 \leq 7$ and thus $\sum_{t=1}^{\infty} n_{t}(\gamma) \leq s-2 \leq 5$. From this and (a) we get that $\kappa(\gamma) \leq \frac{1}{3}+\frac{2}{9}+\frac{2}{27}=\frac{17}{27}$. As $\frac{17}{27}+\frac{1}{3}=\frac{26}{27}<1$, from the last sentence and (3), we get that we have $\kappa(\tilde{\gamma})<1$ for all $\tilde{\gamma} \in \Delta$. Thus $\kappa(\pi)<1$ and therefore (d) holds.

Example 4.4. - We assume that $p \in\{2,3\}$ and that there exists an integer $a \geq 2$ such that we have a ring monomorphism $\mathbb{F}_{p^{a}} \hookrightarrow \operatorname{End}(D[p])$ with the property that $\mathbb{F}_{p^{a}}$ acts on the tangent space of $D[p]$ via scalar endomorphisms. We will check that $\kappa(D)<1$.

To the product decomposition $\mathbb{F}_{p^{a}} \otimes_{\mathbb{F}_{p}} k=k^{a}$ corresponds a direct sum decomposition $M / p M=\oplus_{u=1}^{a} \bar{M}_{u}$. It is easy to see that we can assume that the last sum decomposition is the reduction modulo $p$ of a direct sum decomposition $M=\oplus_{u=1}^{a} M_{u}$ with the property that $\sigma_{\pi}\left(M_{u}\right)=M_{u+1}$, where $M_{a+1}:=M_{1}$. The direct sum decomposition of $W(k)$-modules $\operatorname{End}(M)=\oplus_{u=1}^{a} \oplus_{\tilde{u}=1}^{a} \operatorname{Hom}\left(M_{u}, M_{\tilde{u}}\right)$ allows us to view each $\operatorname{Hom}\left(M_{u}, M_{\tilde{u}}\right)$ as a $W(k)$-submodule of $\operatorname{End}(M)$.

As $\mathbb{F}_{p^{a}}$ acts on the tangent space of $D[p]$ via scalar endomorphisms, we can choose the indexing of $\bar{M}_{u}$ 's such that we have $\bar{F}^{1} \subseteq \bar{M}_{1}$. Thus we can assume that $F^{1} \subseteq M_{1}$. We can also assume that certain subsets of $\left\{e_{1}, \ldots, e_{r}\right\}$ are $W(k)$-bases for the $M_{u}$ 's. From the last two sentences, we get that:

$\left(^{*}\right)$ if $(i, j) \in \mathscr{J}_{+}$, then $e_{i, j} \in \operatorname{Hom}\left(M, F^{1}\right) \subseteq \operatorname{Hom}\left(M, M_{1}\right)$.

Let $\gamma=\left(i_{1}, \ldots, i_{s}\right) \in \Gamma \cup \Delta$. To check that $\kappa(D)<1$, it suffices to show that $\kappa(\gamma)<1$. Based on (3), to check this it suffices to show that if $\gamma \in \Gamma$, then we have $\kappa(\gamma)<1-\frac{1}{p}$. We can assume that $s \geq 3$. As $\gamma \in \Gamma$, we have $\left(i_{s-1}, i_{s}\right) \in \mathscr{J}_{+, 2}$ and $\left(i_{1}, i_{2}\right), \ldots,\left(i_{s-2}, i_{s-1}\right) \in \mathscr{J}_{0,0}$. From this and $\left(^{*}\right)$ we easily get that we have $e_{i_{1}, i_{2}}, \ldots, e_{i_{s-2}, i_{s-1}} \in \operatorname{End}\left(M_{1}\right)$. As $\sigma_{\pi}\left(\operatorname{End}\left(M_{u}\right)\right)=\operatorname{End}\left(M_{u+1}\right)$, we get that each positive integer $i$ such that $e_{\pi^{i}\left(i_{1}\right), \pi^{i}\left(i_{2}\right)} \in \operatorname{End}\left(M_{1}\right)$ is a multiple of $a$. From this and $\left(^{*}\right)$, we get that $\nu\left(i_{1}, i_{2}\right) \subseteq a \mathbb{N}^{*}$. Similarly we argue that $\left\{\nu\left(i_{2}, i_{3}\right), \ldots, \nu\left(i_{s-2}, i_{s-1}\right)\right\} \subseteq a \mathbb{N}^{*}$. Therefore for each $t \in \mathbb{N}^{*} \backslash a \mathbb{N}^{*}$ we have $n_{t}(\gamma)=0$. As in the proof of Lemma 4.3 (a) we argue that for each $t \in \mathbb{N}^{*}$ we have $n_{t a}(\gamma) \leq 2^{t-1}$. Therefore we have $\kappa(\gamma)<\sum_{t=1}^{\infty} 2^{t-1} p^{-a t}=\frac{1}{p^{a}-2}$. As $a \geq 2$, we get that $\kappa(\gamma)<1-\frac{1}{p}$. Thus $\kappa(D)<1$.

REMARK 4.5. - If $p=2$ (resp. $p=3$ ), there exist plenty of examples in which there exist elements $\gamma \in \Gamma_{1}$ (resp. $\gamma \in \Delta_{1}$ ) such that $\kappa(\gamma)>1$ and therefore also $\kappa(\pi)>1$ (for $p=2$ see Example 4.10 below). 


\subsection{Computing $\mathscr{C}_{1}^{0}$ with explicit equations}

Let $\left(h_{1}[1], h_{2}[1], h_{3}[1]\right) \in \mathscr{H}_{1}(k)$. Let $h_{12}[1] \quad:=h_{1}[1] h_{2}[1] \in \mathscr{W}_{+0}(k)$ and $h_{23}[1]:=h_{2}[1] h_{3}[1] \in \mathscr{W}_{0-}(k)$. We have $\left(h_{1}[1], h_{2}[1], h_{3}[1]\right) \in \mathscr{C}_{1}(k)$ if and only if $h_{12}[1]=\sigma_{\phi}\left(h_{23}[1]\right)$, cf. the very definition of the action $\mathbb{T}_{1}$. Writing $h_{12}=1_{M}[1]+$ $\sum_{(i, j) \in \mathscr{J}_{+} \cup \mathscr{J}_{0}} x_{i, j} \bar{e}_{i, j}$ and $h_{23}=1_{M}[1]+\sum_{(i, j) \in \mathscr{J}_{0} \cup \mathscr{J}_{-}} x_{i, j} \bar{e}_{i, j}$ with $x_{i, j}$ as independent variables, the equation $h_{12}[1]=\sigma_{\phi}\left(h_{23}[1]\right)$ gets translated into a system of equations that are of the form $a_{\pi(i), \pi(j)} x_{\pi(i), \pi(j)}=b_{i, j} x_{i, j}^{p}$ with $a_{\pi(i), \pi(j)}, b_{i, j} \in\{0,1\}$ and that are indexed by $(i, j) \in J^{2}$. More precisely, we have $a_{i, j}=1$ (resp. $b_{i, j}=1$ ) if and only if $(i, j) \in \mathscr{J}_{+} \cup \mathscr{J}_{0}$ (resp. $(i, j) \in \mathscr{J}_{0} \cup \mathscr{J}_{-}$). Based on this, by tracing those variables $x_{i, j}$ that can take independently an infinite number of values in $k$, one easily gets that we have $\left(h_{1}[1], h_{2}[1], h_{3}[1]\right) \in \mathscr{C}_{1}^{0}(k)$ if and only if the following three identities hold (to be compared with [25], Subsection 2.3, Formulas (4a) to (4c)):

$$
\begin{aligned}
& h_{12}[1]=1_{M}[1]+\sum_{(i, j) \in \mathscr{J}_{-, 1}} \sum_{\ell=1}^{\nu(i, j)} x_{i, j}^{p^{\ell}} \bar{e}_{\pi^{\ell}(i), \pi^{\ell}(j)}, \\
& h_{2}[1]=1_{M}[1]+\sum_{(i, j) \in \mathscr{J}_{-, 1}} \sum_{\ell=1}^{\nu(i, j)-1} x_{i, j}^{p^{\ell}} \bar{e}_{\pi^{\ell}(i), \pi^{\ell}(j)}, \\
& h_{23}[1]=1_{M}[1]+\sum_{(i, j) \in \mathscr{J}_{-, 1}} \sum_{\ell=0}^{\nu(i, j)-1} x_{i, j}^{p^{\ell}} \bar{e}_{\pi^{\ell}(i), \pi^{\ell}(j)},
\end{aligned}
$$

where the variables $x_{i, j}$ with $(i, j) \in \mathscr{J}_{-, 1}$ can take independently all values in $k$ such that $h_{2}[1] \in \mathscr{W}_{0}(k)$.

Note that Formulas (4), (5), and (6) differ only by summation limits. If $\mathfrak{n}_{0,0}$ is as in Lemma 4.1 (b), then we have $h_{2}[1] \in 1_{M}[1]+\mathfrak{n}_{0,0}$. From this and the fact that $\mathfrak{n}_{0,0}$ is a nilpotent algebra, we get that:

(*) The element $h_{2}[1]$ has an inverse in $1_{M}[1]+\mathfrak{n}_{0,0}$ which is a polynomial in $h_{2}[1]$ with coefficients in $k$ and therefore we always have $h_{2}[1] \in \mathscr{W}_{0}(k)$.

Thus the variables $x_{i, j}$ with $(i, j) \in \mathscr{J}_{-, 1}$ can take independently all values in $k$. Based on Formulas (4) to (6) we get that

$$
\operatorname{Lie}\left(\mathscr{C}_{1}^{0}\right)=\bigoplus_{(i, j) \in \mathscr{J}_{-, 1}} k \bar{e}_{i, j}=\mathfrak{n}_{-, 1} \subseteq \operatorname{Lie}\left(\mathscr{W}_{-, k}\right) .
$$

As $\mathfrak{n}_{-, 1}$ does not contain non-zero semisimple elements, from (7) we get that $\mathbb{G}_{m}$ is not a subgroup of $\mathscr{C}_{1}^{0}$ and thus $\mathscr{C}_{1}^{0}$ is unipotent.

Let $\mathscr{V}_{-1}, \mathscr{V}_{1}$, and $\mathscr{V}_{2}$ be the vector group schemes over $k$ whose Lie algebras are $\mathfrak{n}_{-, 1}$, $\mathfrak{n}_{+, 1}$ and $\mathfrak{n}_{+, 2}$ (respectively). For a commutative $k$-algebra $R$ we have $\mathscr{V}_{-1}(R)=\mathfrak{n}_{-, 1} \otimes_{k} R$, $\mathscr{V}_{1}(R)=\mathfrak{n}_{+, 1} \otimes_{k} R$, and $\mathscr{V}_{2}(R)=\mathfrak{n}_{+, 2} \otimes_{k} R$. We get that the morphism of smooth $k$-schemes

$$
\mathscr{V}_{-1} \rightarrow \mathscr{C}_{1}^{0}
$$

that takes the element $\sum_{(i, j) \in \mathscr{J}_{-, 1}} x_{i, j} \bar{e}_{i, j} \in \mathfrak{n}_{-, 1}=\operatorname{Lie}\left(\mathscr{C}_{1}^{0}\right)=\operatorname{Lie}\left(\mathscr{V}_{-1}\right)=\mathscr{V}_{-1}(k)$ to the element

$$
\left(h_{12}[1]\left(h_{2}[1]\right)^{-1}, h_{2}[1],\left(h_{2}[1]\right)^{-1} h_{23}[1]\right) \in \mathscr{C}_{1}^{0}(k)
$$


obtained naturally from Formulas (4) to (6), is an isomorphism. For $s \in\{1,2\}$ we consider the closed embedding monomorphism

$$
\varepsilon_{s}: \mathscr{V}_{s} \hookrightarrow \mathscr{W}_{+, k}
$$

that takes $v_{s} \in \mathscr{V}_{s}(R)$ to $1_{M / p M \otimes_{k} R}+v_{s} \in \mathscr{W}_{+, k}(R)$.

\subsection{The key morphism $\zeta$}

Let $\mathscr{I}_{1}:=\mathscr{H}_{1} / \mathscr{W}_{-, k}$; it is an affine, smooth group scheme over $k$ isomorphic to $\mathscr{W}_{+0, k}$ and therefore such that we have a short exact sequence

$$
1 \rightarrow \mathscr{W}_{+, k} \rightarrow \mathscr{I}_{1} \rightarrow \mathscr{W}_{0, k} \rightarrow 1
$$

As schemes over $k$ we can identify naturally $\mathscr{I}_{1}=\mathscr{W}_{+, k} \times_{k} \mathscr{W}_{0, k}$. Thus we will also identify (as sets) $\mathscr{I}_{1}(k)=\mathscr{W}_{+}(k) \times \mathscr{W}_{0}(k)$. Let $\epsilon: \mathscr{H}_{1} \rightarrow \mathscr{I}_{1}$ and $\theta: \mathscr{I}_{1} \rightarrow \mathscr{W}_{0, k}$ be the natural epimorphisms and let $\iota:=\theta \circ \epsilon: \mathscr{H}_{1} \rightarrow \mathscr{W}_{0, k}$ be their composite. The epimorphism $\epsilon$ gives birth via restriction to a finite homomorphism $\mathscr{C}_{1}^{0} \rightarrow \mathscr{I}_{1}$ whose kernel is finite and connected.

The group $\tilde{\mathscr{C}}_{1}^{0}:=\operatorname{Im}\left(\mathscr{C}_{1}^{0} \rightarrow \mathscr{I}_{1}\right)$ is isomorphic to $\mathscr{C}_{1}^{0}$. More precisely, using the isomorphism $\mathscr{V}_{-1} \rightarrow \mathscr{C}_{1}^{0}$ one easily gets that the epimorphism $\mathscr{C}_{1}^{0} \rightarrow \tilde{\mathscr{C}}_{1}^{0}$ can be identified with the Frobenius endomorphism of $\mathscr{C}_{1}^{0}$. We have

$$
\operatorname{Lie}\left(\tilde{\mathscr{C}}_{1}^{0}\right)=\bigoplus_{(i, j) \in \mathscr{J}_{0,0} \cup \mathscr{J}_{+, 1}, \eta(i, j)=1} k \bar{e}_{i, j} .
$$

Definition 4.6. - The morphism $\zeta: \tilde{\mathscr{C}}_{1}^{0} \times_{k} \mathscr{V}_{2} \times_{k} \mathscr{W}_{0, k} \rightarrow \mathscr{I}_{1}$ of $k$-schemes is defined by the following rule: if $R$ is a commutative $k$-algebra, then the element $(\tilde{h}, y, z) \in$ $\tilde{\mathscr{C}}_{1}^{0}(R) \times \mathscr{V}_{2}(R) \times \mathscr{W}_{0, k}(R)$ is mapped to the product element $\tilde{h} \cdot\left(\varepsilon_{2}(y), 1\right) \cdot \epsilon(1, z, 1) \in \mathscr{I}_{1}(R)$.

The key step for proving the main result Theorem 1.2 is to show that $\zeta$ is finite and flat. For this, we first prove the following basic fact.

Lemma 4.7. - Let $R$ be a commutative $\mathbb{F}_{p}$-algebra. Let $n \in \mathbb{N}^{*}$. Let $d_{1}, \ldots, d_{n}$ be positive integers. For each $\ell \in\{1, \ldots, n\}$ we consider a polynomial $Q_{\ell} \in R\left[x_{1}, \ldots, x_{n}\right]$. We assume that there exist positive rational numbers $\mu_{1}, \ldots, \mu_{n}$ such that for each $\ell \in\{1, \ldots, n\}$ and for every monomial term $\beta \prod_{i=1}^{n} x_{i}^{v_{i}}$ of $Q_{\ell}$ with $\beta \in R \backslash\{0\}$ we have an inequality $\mu_{\ell} d_{\ell}>\sum_{i=1}^{n} \mu_{i} v_{i}$ (thus the degree of each $Q_{\ell}$ in the variable $x_{\ell}$ is at most equal to $d_{\ell}-1$ ). Let

$$
\mathscr{R}:=R\left[x_{1}, \ldots, x_{n}\right] /\left(x_{1}^{d_{1}}-Q_{1}, \ldots, x_{n}^{d_{n}}-Q_{n}\right) .
$$

Then $\mathscr{R}$ is a free $R$-module of rank $\prod_{\ell=1}^{n} d_{\ell}$. Therefore the natural morphism $\operatorname{Spec} \mathscr{R} \rightarrow \operatorname{Spec} R$ of schemes is a finite, flat cover of degree $\prod_{\ell=1}^{n} d_{\ell}$.

Proof. - Multiplying all $\mu_{1}, \ldots, \mu_{n}$ by a positive integer, we can assume that we have $\mu_{1}, \ldots, \mu_{n} \in \mathbb{N}^{*}$. Let $\mathbb{M}:=\mathbb{N}^{d}$. Let $\tau: \mathbb{M} \hookrightarrow \mathbb{M}$ be the monomorphism of additive monoids that takes a sequence $\left(v_{1}, \ldots, v_{n}\right) \in \mathbb{M}$ to $\left(\mu_{1} v_{1}, \ldots, \mu_{n} v_{n}\right) \in \mathbb{M}$. Let $<$ be the degree-lexicographic ordering on $\mathbb{M}$. Let $\prec$ be the well ordering on $\mathbb{M}$ such that for $a, b \in \mathbb{M}$ we have $a \prec b$ if and only if $\tau(a)<\tau(b)$. For each $P \in R\left[x_{1}, \ldots, x_{n}\right]$, we define its weight $\omega(P)$ to be the maximal element $\left(v_{1}, \ldots, v_{n}\right)$ of $\mathbb{M}$ with respect to the ordering $\prec$ for which the monomial $\prod_{i=1}^{n} x_{i}^{v_{i}}$ appears in $P$ with a non-zero coefficient. From hypotheses 
we get that for each $\ell \in\{1, \ldots, n\}$ we have $\omega\left(x_{\ell}^{d_{\ell}}\right)>\omega\left(Q_{\ell}\right)$. Using this, it is an easy exercise in the theory of Gröbner bases (over the base $R$ ) to check that the image of the set $\left\{\prod_{i=1}^{n} x_{i}^{v_{i}} \mid 0 \leq v_{i}<d_{i} \forall i \in\{1, \ldots, n\}\right\}$ in $\mathscr{R}$ is an $R$-basis for $\mathscr{R}$. From this the lemma follows.

THeOREM 4.8. - We assume that we have an inequality $\kappa(D[p])<1$. Then the morphism $\zeta$ is a finite, flat morphism of degree $p^{\left|\mathscr{J}_{0,0}\right|}$.

Proof. - Each element of $\mathscr{W}_{+}(k)$ can be written uniquely as $\varepsilon_{1}\left(a_{1}\right) \varepsilon_{2}\left(a_{2}\right)$ with $a_{1} \in \mathfrak{n}_{+, 1}$ and $a_{2} \in \mathfrak{n}_{+, 2}$. Thus each element of $\mathscr{I}_{1}(k)$ can be written uniquely as a pair $\left(\varepsilon_{1}\left(a_{1}\right) \varepsilon_{2}\left(a_{2}\right), a_{0}\right)$ with $a_{0} \in \mathscr{W}_{0}(k)$. Let $(\tilde{h}, y, z) \in \tilde{\mathscr{C}}_{1}^{0}(k) \times \mathscr{V}_{2}(k) \times \mathscr{W}_{0, k}(k)$ be an arbitrary element. We look at the equation

$$
\zeta((\tilde{h}, y, z))=\left(\varepsilon_{1}\left(a_{1}\right) \varepsilon_{2}\left(a_{2}\right), a_{0}\right) .
$$

By applying $\theta$ to (8) we get that

$$
\iota(\tilde{h}) z=a_{0} .
$$

We write $\tilde{h}=\left(h_{1}(x), h_{2}(x)\right)$ with $x \in \operatorname{Lie}\left(\tilde{\mathscr{C}}_{1}^{0}\right)$. We have $h_{2}(x) z=a_{0}$, cf. (9).

We write $y=\sum_{(i, j) \in \mathscr{J}_{+, 2}} y_{i, j} \bar{e}_{i, j}$ and $x=\sum_{(i, j) \in \mathscr{J}_{0,0} \cup \mathscr{J}_{+, 1}, \eta(i, j)=1} x_{i, j} \bar{e}_{i, j}$, where $y_{i, j}$ 's and $x_{i, j}$ 's are variables. Based on Formulas (4) and (5), we have

$$
h_{2}(x)=1_{M}[1]+\sum_{(i, j) \in \mathscr{J}_{0,0}, \eta(i, j)=1} \sum_{l=0}^{\nu(i, j)-1} x_{i, j}^{p^{l}} \bar{e}_{\pi^{l}(i), \pi^{l}(j)}
$$

and

$$
h_{12}(x):=h_{1}(x) h_{2}(x)=1_{M}[1]+\sum_{(i, j) \in \mathscr{J}_{0,0} \cup \mathscr{J}_{+, 1}, \eta(i, j)=1} \sum_{l=0}^{\nu(i, j)} x_{i, j}^{p^{l}} \bar{e}_{\pi^{l}(i), \pi^{l}(j)} .
$$

By applying (8) and (9), by using the identification $\mathscr{I}_{1}(k)=\mathscr{W}_{+}(k) \times \mathscr{W}_{0}(k)$, and by denoting with 1 identity elements, we get that

$$
\left(h_{1}(x), h_{2}(x)\right) \cdot(1+y, 1) \cdot(1, z)=\left(1+a_{1}+a_{2}, 1\right) \cdot\left(1, h_{2}(x)\right) \cdot(1, z) .
$$

Thus we get the equation $\left(h_{1}(x), h_{2}(x)\right) \cdot(1+y, 1)=\left(1+a_{1}+a_{2}, 1\right) \cdot\left(1, h_{2}(x)\right)$ between elements of $\mathscr{I}_{1}(k)$ and therefore the equation

$$
h_{12}(x)\left(1_{M}[1]+y\right)=\left(1_{M}[1]+a_{1}+a_{2}\right) h_{2}(x)
$$

between elements of $\mathbf{G L}_{M}(k)$. As $\mathfrak{n}_{+}^{2}=0$, we have $h_{12}(x) y=h_{2}(x) y$. Thus Equation (10) is equivalent to $h_{12}(x)+h_{2}(x) y=h_{2}(x)+\left(a_{1}+a_{2}\right) h_{2}(x)$. Let

$$
l_{1}(x):=h_{12}(x)-h_{2}(x)=\sum_{(i, j) \in \mathscr{J}_{0,0} \cup \mathscr{J}_{+, 1}, \eta(i, j)=1} x_{i, j}^{p^{\nu(i, j)}} \bar{e}_{\pi^{\nu(i, j)}(i), \pi^{\nu(i, j)}(j)} \in \mathfrak{n}_{+, 1}
$$

and

$$
l_{2}(x):=h_{2}(x)-1_{M}[1]=\sum_{(i, j) \in \mathscr{J}_{0,0}, \eta(i, j)=1} \sum_{l=0}^{\nu(i, j)-1} x_{i, j}^{p^{l}} \bar{e}_{\pi^{l}(i), \pi^{l}(j)}=: \sum_{\left(i^{\prime}, j^{\prime}\right) \in \mathscr{J}_{0,0}} q_{i^{\prime}, j^{\prime}}(x) \bar{e}_{i^{\prime}, j^{\prime}} .
$$

We get that Equation (10) can be rewritten as

$$
l_{1}(x)+h_{2}(x) y=\left(a_{1}+a_{2}\right) h_{2}(x) .
$$

$4^{\mathrm{e}}$ SÉRIE - TOME $43-2010-\mathrm{N}^{\mathrm{o}} 6$ 
For $s \in\{1,2\}$ let

$$
\pi_{s}: \operatorname{Lie}\left(\mathscr{W}_{+, k}\right) \rightarrow \operatorname{Lie}\left(\mathscr{V}_{s}\right)
$$

be the projection of $\operatorname{Lie}\left(\mathscr{W}_{+, k}\right)$ on $\operatorname{Lie}\left(\mathscr{V}_{s}\right)$ along $\operatorname{Lie}\left(\mathscr{V}_{3-s}\right)$. Multiplying (11) from the left by $h_{2}(x)^{-1}$ and using that $h_{2}(x)^{-1} l_{1}(x)=\sum_{s=0}^{\infty}(-1)^{s} l_{2}(x)^{s} l_{1}(x) \in \mathfrak{n}_{+, 1}$ and $h_{2}(x)^{-1} a_{1} h_{2}(x) \in \mathfrak{n}_{+, 1}$ (cf. Property $4.3(*)$ and Lemma 4.1 (c)), we get that

$$
y=\pi_{2}\left(h_{2}(x)^{-1} a_{2} h_{2}(x)\right) .
$$

Due to (12), solving Equation (11) is the same thing as solving the equation $h_{2}(x)^{-1} l_{1}(x)=$ $\pi_{1}\left(h_{2}(x)^{-1}\left(a_{1}+a_{2}\right) h_{2}(x)\right)$ and therefore the equation

$$
\begin{aligned}
& l_{1}(x)=\left[1_{M}[1]+l_{2}(x)\right] \pi_{1}\left(\sum_{s=0}^{\infty}(-1)^{s} l_{2}(x)^{s}\left(a_{1}+a_{2}\right)\left[1_{M}[1]+l_{2}(x)\right]\right)= \\
& a_{1}+a_{1} l_{2}(x)+\left[1_{M}[1]+l_{2}(x)\right] \pi_{1}\left(\sum_{s=0}^{\infty}(-1)^{s} l_{2}(x)^{s}\left(a_{2}\right)\left[1_{M}[1]+l_{2}(x)\right]\right) .
\end{aligned}
$$

The last identity is implied by Lemma 4.1 (c).

We write $a_{1}+a_{2}=\sum_{(i, j) \in \mathscr{J}_{+}} a_{i, j} \bar{e}_{i, j}$, with the $a_{i, j}$ 's thought as variables. We consider the polynomial $k$-algebra $R:=k\left[a_{i, j} \mid(i, j) \in \mathscr{J}_{+}\right]$. Due to the above formulas for $l_{1}(x)$ and $l_{2}(x)$, the system of equations defined by Equation (13) has the form

$$
x_{i, j}^{p^{\nu(i, j)}}=Q_{i, j} \text { for }(i, j) \in \mathscr{J}_{0,0} \cup \mathscr{J}_{+, 1} \text { with } \eta(i, j)=1,
$$

where each $Q_{i, j}$ is a polynomial in the variables $x_{i, j}$ 's with coefficients in $R$. This system defines a morphism $\operatorname{Spec} \mathscr{R} \rightarrow \operatorname{Spec} R$ of affine $k$-schemes. For $(i, j) \in \mathscr{J}_{0,0} \cup \mathscr{J}_{+, 1}$ with $\eta(i, j)=1$, by identifying the coefficients of $\bar{e}_{\pi^{\nu(i, j)}(i), \pi^{\nu(i, j)}(j)}$ in the two sides of Equation (13), we get the formula:

$$
\begin{aligned}
& Q_{i, j}= \sum_{\substack{\left(i_{1}^{\prime}, i_{2}^{\prime}, \ldots, i_{s-1}^{\prime}, i_{s}^{\prime}\right) \in \Gamma_{1} \\
i_{1}^{\prime}=\pi^{\nu(i, j)}(i) \\
i_{s}^{\prime}=\pi^{\nu(i, j)}(j)}}(-1)^{s-2} a_{i_{s-1}^{\prime}, i_{s}^{\prime}} q_{i_{1}^{\prime}, i_{2}^{\prime}}(x) \cdots q_{i_{s-2}^{\prime}, i_{s-1}^{\prime}}(x) \\
&+\sum_{\substack{\left(i_{1}^{\prime}, i_{2}^{\prime}, \ldots, i_{s-1}^{\prime}, i_{s}^{\prime}\right) \in \Delta_{1} \\
i_{1}^{\prime}=\pi^{\nu(i, j)}(i) \\
i_{s}^{\prime}=\pi^{\nu(i, j)}(j)}}(-1)^{s-3} a_{i_{s-2}^{\prime}, i_{s-1}^{\prime}} q_{i_{1}^{\prime}, i_{2}^{\prime}}(x) \cdots q_{i_{s-3}^{\prime}, i_{s-2}^{\prime}}(x) q_{i_{s-1}^{\prime}, i_{s}^{\prime}}(x) \\
&+a_{\pi^{\nu(i, j)}(i), \pi^{\nu(i, j)}(j)}+\sum_{\substack{i^{\prime} \in\{1, \ldots, r\} \\
\left(\pi^{\nu(i, j)}(i), i^{\prime}\right) \in \mathscr{J}_{+, 1} \\
\left(i^{\prime}, \pi^{\nu(i, j)}(j)\right) \in \mathscr{J}_{0,0}}} a_{\pi^{\nu(i, j)}(i), i^{\prime}} q_{i^{\prime}, \pi^{\nu(i, j)}(j)}(x) .
\end{aligned}
$$

In Formula (15), the first two lines record (resp. the third line records) the contribution of $a_{2}$ (resp. of $a_{1}$ ) to $Q_{i, j}$.

Let $n$ be the number of elements of $\mathscr{J}_{-, 1}$. We write

$$
\left\{(i, j) \in \mathscr{J}_{0,0} \cup \mathscr{J}_{+, 1} \mid \eta(i, j)=1\right\}=\left\{\left(i_{1}, j_{1}\right), \ldots,\left(i_{n}, j_{n}\right)\right\} .
$$

We can assume that $\kappa(\pi)<1$, cf. the very definition of $\kappa(D[p])$. Let

$$
\nu:=\max \left\{\nu\left(i_{\ell}, j_{\ell}\right) \mid \ell \in\{1, \ldots, n\}\right\} .
$$


For each $\ell \in\{1, \ldots, n\}$ we define a positive integer (thought as a weight) $\mu_{\ell}:=p^{\nu-\nu\left(i_{\ell}, j_{\ell}\right)}$. We have $\mu_{\ell} p^{\nu\left(i_{\ell}, j_{\ell}\right)}=p^{\nu}$.

We consider a monomial term:

$$
\beta \prod_{\ell=1}^{n} x_{i_{\ell}, j_{\ell}}^{t_{\ell}}=(-1)^{s-2} a_{i_{s-1}^{\prime}, i_{s}^{\prime}} q_{i_{1}^{\prime}, i_{2}^{\prime}}(x) \cdots q_{i_{s-2}^{\prime}, i_{s-1}^{\prime}}(x)
$$

with $\gamma:=\left(i_{1}^{\prime}, i_{2}^{\prime}, \ldots, i_{s-1}^{\prime}, i_{s}^{\prime}\right) \in \Gamma_{1}$ (resp.

$$
\beta \prod_{\ell=1}^{n} x_{i_{\ell}, j_{\ell}}^{t_{\ell}}=(-1)^{s-3} a_{i_{s-2}^{\prime}, i_{s-1}^{\prime}} q_{i_{1}^{\prime}, i_{2}^{\prime}}(x) \cdots q_{i_{s-3}^{\prime}, i_{s-2}^{\prime}}(x) q_{i_{s-1}^{\prime}, i_{s}^{\prime}}(x)
$$

with $\left.\gamma:=\left(i_{1}^{\prime}, i_{2}^{\prime}, \ldots, i_{s-1}^{\prime}, i_{s}^{\prime}\right) \in \Delta_{1}\right)$ that contributes via Equation (15) to some $Q_{i_{a}, j_{a}}$. If $u \in\{1, \ldots, s-1\}$ is such that $\left(i_{u}^{\prime}, i_{u+1}^{\prime}\right) \in \mathscr{J}_{0,0}$, then for

$$
\left(i_{\ell}, j_{\ell}\right):=\pi^{-\eta\left(i_{u}^{\prime}, i_{u+1}^{\prime}\right)+1}\left(i_{u}^{\prime}, i_{u+1}^{\prime}\right) \in\left\{(i, j) \in \mathscr{J}_{0,0} \cup \mathscr{J}_{+, 1} \mid \eta(i, j)=1\right\}
$$

with $\ell \in\{1, \ldots, n\}$ we get that the contribution of $x_{i_{\ell}, j_{\ell}}$ to $\beta \prod_{\ell=1}^{n} x_{i_{\ell}, j_{\ell}}^{t_{\ell}}$ that corresponds to

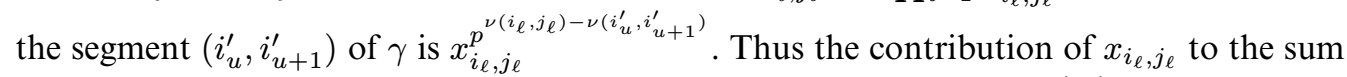
$\sum_{\ell=1}^{n} t_{\ell} \mu_{\ell}$ that corresponds to the segment $\left(i_{u}^{\prime}, i_{u+1}^{\prime}\right)$ is precisely $p^{\nu-\nu\left(i_{u}^{\prime}, i_{u+1}^{\prime}\right)}$. For $t \in \mathbb{N}^{*}$, let $n_{t}(\gamma)$ be as in Subsection 4.2 (i.e., the number of those $u \in\{1, \ldots, s-1\}$ such that we have $\left(i_{u}^{\prime}, i_{u+1}^{\prime}\right) \in \mathscr{J}_{0,0}$ and $\nu\left(i_{u}^{\prime}, i_{u+1}^{\prime}\right)=t$ ). From the last two sentences, we get that

$$
\sum_{\ell=1}^{n} t_{\ell} \mu_{\ell}=p^{\nu} \sum_{t=1}^{\infty} n_{t}(\gamma) p^{-t}=p^{\nu} \kappa(\gamma) \leq p^{\nu} \kappa(\pi)<p^{\nu}
$$

(the first inequality, cf. the very definition of $\kappa(\pi)$ ).

If $\beta \prod_{\ell=1}^{n} x_{i_{\ell}, j_{\ell}}^{t_{\ell}}$ is $a_{\pi^{\nu(i, j)}(i), \pi^{\nu(i, j)}(j)} \quad$ (resp. is $a_{\pi^{\nu(i, j)}(i), i^{\prime}} q_{i^{\prime}, \pi^{\nu(i, j)}(j)}(x)$ with $\left(\pi^{\nu(i, j)}(i), i^{\prime}\right) \in \mathscr{J}_{+, 1}$ and $\left.\left(i^{\prime}, \pi^{\nu(i, j)}(j)\right) \in \mathscr{J}_{0,0}\right)$, then the sum $\sum_{\ell=1}^{n} t_{\ell} \mu_{\ell}$ is 0 (resp. is $\left.p^{\nu-\nu\left(i^{\prime}, \pi^{\nu(i, j)}(j)\right)} \leq p^{\nu-1}\right)$ and thus it is less than $p^{\nu}$.

From the last two paragraphs we get that for every monomial term $\beta \prod_{\ell=1}^{n} x_{i_{\ell}, j_{\ell}}^{t_{\ell}}$ that shows up with a non-zero coefficient in some $Q_{i_{a}, j_{a}}$ with $a \in\{1, \ldots, n\}$, we have $\sum_{\ell=1}^{n} t_{\ell} \mu_{\ell}<p^{\nu}$ and thus (cf. (14)) the hypotheses of Lemma 4.7 are satisfied. Thus from Lemma 4.7 we get that the morphism $\operatorname{Spec} \mathscr{R} \rightarrow \operatorname{Spec} R$ is a finite, flat cover of degree $\prod_{\ell=1}^{n} p^{\nu\left(i_{\ell}, j_{\ell}\right)}=p^{\sum_{(i, j) \in \mathscr{J}_{-, 1}} \nu(i, j)-1}=p^{\left|\mathscr{J}_{0,0}\right|}$.

All the above part can be redone using arbitrary $\mathscr{K}$-valued points instead of $k$-valued points. Taking $\mathscr{K}$ to be the $k$-algebra of global functions of the affine $k$-scheme $\mathscr{I}_{1}$, from Equation (9) and the fact that the morphism $\operatorname{Spec} \mathscr{R} \rightarrow \operatorname{Spec} R$ is a finite, flat cover of degree $p^{\left|\mathscr{J}_{0,0}\right|}$, we get that $\zeta$ is a finite, flat cover of degree $p^{\left|\mathscr{J}_{0,0}\right|}$.

\subsection{Examples}

Example 4.9. - Suppose that $D$ is minimal, i.e. we have $n_{D}=1$. As $n_{D}=1$, we can assume that $g_{\pi}=1_{M}$ and thus that $\sigma_{\phi}=\sigma_{\pi}$. As $\sigma_{\phi}=\sigma_{\pi}$, we have direct sum decompositions:

$$
(\operatorname{End}(M), \phi)=\oplus_{\alpha \in \mathbb{Q} \cap[-1,1]}\left(W_{\alpha}, \phi\right)=\left(S_{+}, \phi\right) \oplus\left(S_{0}, \phi\right) \oplus\left(S_{-}, \phi\right)
$$


such that all Newton polygon slopes of $\left(W_{\alpha}, \phi\right)$ are $\alpha$, all Newton polygon slopes of $\left(S_{+}, \phi\right)$ are positive, all Newton polygon slopes of $\left(S_{0}, \phi\right)$ are 0 , and all Newton polygon slopes of $\left(S_{-}, \phi\right)$ are negative.

Referring to Equation (11), in this paragraph we will check that $y, a_{2} \in S_{+} / p S_{+}$. To check this we can assume that either $D$ is indecomposable or a direct sum of two indecomposable minimal $p$-divisible groups over $k$ that have distinct Newton polygon slopes. In the first case we have $S_{+}=S_{-}=0$ and from [25], Example 2.3.1, we get that $\mathscr{J}_{-, 2}=\mathscr{J}_{-} \backslash \mathscr{J}_{-, 1}=\varnothing$ and therefore that $\mathscr{J}_{+, 1}=\mathscr{J}_{+}$and $\mathscr{J}_{+, 2}=\varnothing$; thus $y=a_{2}=0 \in S_{+} / p S_{+}$. In the second case, we write $D=D_{1} \times D_{2}$ such that both $D_{1}$ and $D_{2}$ are indecomposable minimal $p$-divisible groups over $k$. For $s \in\{1,2\}$, let $c_{s}$ and $d_{s}$ be the codimension and the dimension of $D_{s}$. We can assume that the Newton polygon slope $\frac{d_{1}}{c_{1}+d_{1}}$ of $D_{1}$ is less than the Newton polygon slope $\frac{d_{2}}{c_{2}+d_{2}}$ of $D_{2}$. We have $c=c_{1}+c_{2}, d=d_{1}+d_{2}$, and $c_{2} d_{1}<c_{1} d_{2}$. Let $(M, \phi)=\left(M_{1}, \phi\right) \oplus\left(M_{2}, \phi\right)$ be the direct sum decomposition such that the Dieudonné module of $D_{s}$ is $\left(M_{s}, \phi\right)$. We have $S_{+}=\operatorname{Hom}\left(M_{1}, M_{2}\right), S_{0}=\operatorname{End}\left(M_{1}\right) \oplus \operatorname{End}\left(M_{2}\right)$, and $S_{-}=\operatorname{Hom}\left(M_{2}, M_{1}\right)$. From [25], Example 2.3.2, we get that if $(i, j) \in \mathscr{J}_{-}$is such that $e_{i, j} \in \operatorname{Hom}\left(M_{1}, M_{2}\right)$, then we have $(i, j) \in \mathscr{J}_{-, 1}$. The $W(k)$-linear map that takes $e_{i, j}$ to $e_{j, i}$ induces naturally an isomorphism between $\left(\operatorname{Hom}\left(M_{2}, M_{1}\right), \phi\right)$ and the dual of $\left(\operatorname{Hom}\left(M_{1}, M_{2}, \phi\right)\right)$ (in the natural sense that involves no Tate twists). From the previous two sentences we get via duality that if $(i, j) \in \mathscr{J}_{+}$is such that $e_{i, j} \in \operatorname{Hom}\left(M_{2}, M_{1}\right)$, then we have $(i, j) \in \mathscr{J}_{+, 1}$. From this and the first case, we get that if $(i, j) \in \mathscr{J}_{+, 2}$, then we have $e_{i, j} \in \operatorname{Hom}\left(M_{1}, M_{2}\right)=S_{+}$. This implies that $y, a_{2} \in S_{+} / p S_{+}$.

In this paragraph we assume that $a_{1}=0$. As $y, a_{2} \in S_{+} / p S_{+}$and $a_{1}=0$, from Equation (11) we get by an increasing induction on the Newton polygon slope $\alpha \in \mathbb{Q} \cap[-1,1]$ that the component of $x$ in $W_{\alpha} / p W_{\alpha}$ is uniquely determined. Thus the system (11) has for $a_{1}=0 \mathrm{a}$ unique solution. More precisely, for $a_{1}=0$ the system (13) is of the form:

$$
x_{1}^{v_{1}}=a_{1}, \quad x_{2}^{v_{2}}=Q_{2}\left(x_{1}\right), \quad \cdots, \quad x_{n}^{v_{n}}=Q_{n}\left(x_{1}, \ldots, x_{n-1}\right),
$$

where $\left\{x_{1}, \ldots, x_{n}\right\}=\left\{x_{i_{1}, j_{1}}, \ldots, x_{i_{n}, j_{n}}\right\}$, where $v_{1}, \ldots, v_{n}$ are the corresponding rearrangement of $\nu_{i_{1}, j_{1}}, \ldots, \nu_{i_{n}, j_{n}}$, and where $a_{1}, Q_{2}, \ldots, Q_{n}$ are the corresponding rearrangement of $Q_{i_{1}, j_{1}}, Q_{i_{2}, j_{2}}, \ldots, Q_{i_{n}, j_{n}}$. Thus, if $I$ is the ideal of $R$ generated by $a_{i, j}$ with $(i, j) \in \mathscr{J}_{+, 1}$, then the morphism Spec $\mathscr{R} / \mathscr{R} I \rightarrow \operatorname{Spec} R / I$ is a purely inseparable, finite, flat cover of degree $p^{\left|\mathscr{J}_{0,0}\right|}$.

If $D$ is isoclinic, then $\mathscr{J}_{+, 2}=\varnothing, \kappa(D)=0$, and the condition (C) holds. Thus the morphism Spec $\mathscr{R} \rightarrow \operatorname{Spec} R$ is a finite, flat cover of degree $p^{\left|\mathscr{J}_{0,0}\right|}$.

EXAmPle 4.10. - Suppose that $\pi$ is the $r$-cycle $(12 \cdots r)$. We compute several invariants introduced above in this section. We have:

$$
\begin{aligned}
\mathscr{J}_{-, 1} & =\{(i, r) \mid i \leq c\} \cup\{(c, j) \mid c+1 \leq j \leq r-1\}, \\
\mathscr{J}_{+, 1} & =\{(c+1, j) \mid j \leq c\} \cup\{(i, 1) \mid c+2 \leq i \leq r\}, \\
\mathscr{J}_{0,0} & =\{(i, j) \mid r \geq j>i>c\} \cup\{(i, j) \mid c>i>j \geq 1\} .
\end{aligned}
$$

- Here is a pictorial representation of the sets for $c=d=4$. The gray diagonal and the arrow remind how the $r$-cycle $\pi$ acts on the diagram. 


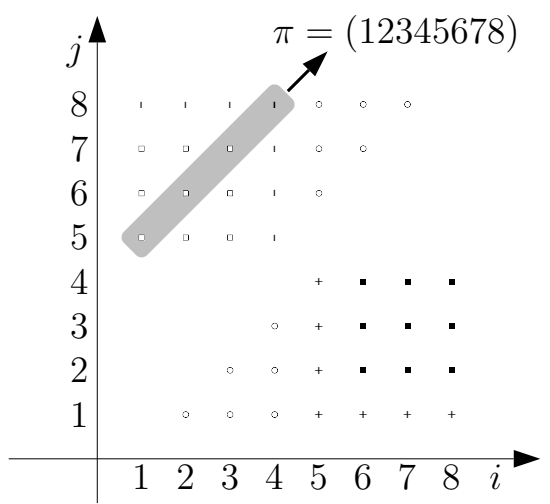

N.B. We write: $\mid \in \mathscr{J}_{-, 1}, o \in \mathscr{J}_{0,0},+\in \mathscr{J}_{+, 1}, \square \in \mathscr{J}_{-, 2}, \mathbf{\square} \in \mathscr{J}_{+, 2}$.

- We compute the numbers: $\nu(i, j)$ with $(i, j) \in \mathscr{J}_{-, 1}$. If $(i, j) \in \mathscr{J}_{-, 1}$ and $j=r$, then $\nu(i, j)=c+1-i$; if $(i, j) \in \mathscr{J}_{-, 1}$ and $i=c$, then $\nu(i, j)=r+1-j$. Note that $\left|\mathscr{J}_{0,0}\right|=\frac{c(c-1)+d(d-1)}{2}$ and that $n=\left|\mathscr{J}_{-, 1}\right|=r-1$. The set of $\pi$-orders of elements of $\mathscr{J}_{-, 1}$ is $\{0,1,2, \ldots, \max \{c, d\}\}$ and therefore $\nu=\max \{c, d\}-1$.

- Note that the nilpotency bounds of Lemma 4.1 (b) and (c) are sharp for $\pi=(12 \cdots r-1 r)$. For example, we have $\mathfrak{n}_{0,0}^{c-1} \neq 0$ as by going down the line $j=i-1$ we have an identity:

$$
\bar{e}_{c, c-1} \bar{e}_{c-1, c-2} \cdots \bar{e}_{2,1}=\bar{e}_{c, 1} .
$$

- We compute the invariant $\kappa(\pi)$ explicitly for $p=2,3$. As $\pi$ is an $r$-cycle, it is easy to see that $\pi$ is unique up to conjugation by a permutation of the set $J$ that leaves invariant the subset $\{1, \ldots, c\}$. Therefore we have $\kappa(D[p])=\kappa(\pi)$. Suppose that $c \geq 3$ and $d \geq 2$. The maximal value of $\kappa(\gamma)$ with $\gamma \in \Gamma_{1} \cup \Delta_{1}$ is obtained for certain elements $\gamma \in \Delta_{1}$. Like for $\gamma=(c+1, c+2, \ldots, r, c, 2)$, we have

$$
\kappa(\gamma)=\frac{2}{p}+\frac{1}{p^{2}}+\frac{1}{p^{3}}+\cdots+\frac{1}{p^{d-1}} .
$$

For $p=3$, clearly $\kappa(\pi)<1$. For $p=2$, we have $\kappa(\pi) \geq 1$. Using this one gets that if $p=2$ and $c=d \geq 3$, then $D$ is isomorphic to $D^{\vee}$ and the condition (C) does not hold for $D$.

- Suppose $p=2$ and $(c, d)=(3,2)$. Then $\mathscr{J}_{+, 1}=\{(4,1),(4,2),(4,3),(5,1)\}, \mathscr{J}_{+, 2}=$ $\{(5,2),(5,3)\}$, and $\mathscr{J}_{0,0}=\{(2,1),(3,1),(3,2),(4,5)\}$.

Thus $\Gamma_{1}=\{(4,5,2),(4,5,3)\}, \quad \Delta_{1}=\{(4,5,3,2),(5,2,1),(5,3,1)\}, \quad$ and $\quad \kappa(\pi)=$ $\kappa(D[p])=1$. The system (14) is of the form (cf. (15))

$$
\begin{gathered}
x_{2,1}^{4}=a_{5,3} x_{4,5}+a_{4,3}, \quad x_{3,1}^{2}=a_{5,2} x_{4,5}+a_{5,3} x_{4,5} x_{2,1}^{2}+a_{4,2}+a_{4,3} x_{2,1}^{2} \\
x_{4,5}^{2}=a_{5,2} x_{2,1}+a_{5,3} x_{3,1}+a_{5,1}, \quad x_{4,1}=a_{4,1}+a_{4,2} x_{2,1}+a_{4,3} x_{3,1} .
\end{gathered}
$$

Eliminating $x_{4,1}$ and substituting $x_{3,1}=t^{7 / 4}$, Lemma 4.7 implies that this system defines a finite morphism (i.e., $\zeta$ is a finite morphism despite the fact that $\kappa(\pi)=$ $\kappa(D[p])=1)$. This points out that the general weights $\mu_{\ell}$ used in the proof of Theorem 4.8 can occasionally be replaced by other weights which can still make the proof of Theorem 4.8 work even if $\kappa(D[p]) \geq 1$. 


\section{Proof of Theorem 1.2}

In this section, we prove Theorem 1.2 (see Subsection 5.2).

\subsection{Exact subgroups}

We first recall from [2] several properties of exact subgroups of an affine, smooth, connected group $\mathscr{G}$ over a field which are needed in Subsection 5.2. A smooth subgroup scheme $\mathscr{F}$ of $\mathscr{G}$ is called exact if the induction of rational $\mathscr{F}$-modules to rational $\mathscr{G}$-modules preserves short exact sequences.

THEOREM 5.1. - The following six properties hold:

(a) The smooth subgroup scheme $\mathscr{F}$ of $\mathscr{G}$ is exact if and only if the quotient variety $\mathscr{G} / \mathscr{F}$ is affine.

(b) The smooth subgroup scheme $\mathscr{F}$ of $\mathscr{G}$ is exact if and only if its identity component $\mathscr{F}^{0}$ is exact.

(c) If $\mathscr{G}$ is unipotent, then each smooth subgroup of it is exact.

(d) If $\mathscr{K}$ is a smooth subgroup of $\mathscr{F}$ which is exact in $\mathscr{F}$ and if $\mathscr{F}$ is exact in $\mathscr{G}$, then $\mathscr{K}$ is exact in $\mathscr{G}$.

(e) Let $\mathscr{N}$ be a normal, smooth, connected subgroup of $\mathscr{G}$. Let $\tilde{\mathscr{F}}:=\operatorname{Im}(\mathscr{F} \rightarrow \mathscr{G} / \mathscr{N})$; it is a smooth subgroup of $\mathscr{G} / \mathscr{N}$. Then $\tilde{\mathscr{F}}$ is exact in $\mathscr{G} / \mathscr{N}$ if and only if the smooth subgroup $\mathscr{N} \mathscr{F}$ is exact in $\mathscr{G}$.

(f) If $\mathscr{F}$ is exact in $\mathscr{G}$, then it is exact in every other smooth, connected subgroup $\mathscr{K}$ of $\mathscr{G}$ that contains $\mathscr{F}$.

Proof. - Part (a) is the main result of [2] (cf. [2], Theorem 4.3). The natural morphism $\mathscr{G} / \mathscr{F}^{0} \rightarrow \mathscr{G} / \mathscr{F}$ is finite and surjective. Thus $\mathscr{G} / \mathscr{F}^{0}$ is an affine variety if and only if $\mathscr{G} / \mathscr{F}$ is an affine variety, cf. Chevalley's theorem of [5], Théorème (6.7.1). From this and (a), we get that (b) holds. Parts (c) and (d) are implied by [2], Corollary 2.2 and [2], Proposition 2.3 (respectively). Part (e) follows from (a) once we remark that $(\mathscr{G} / \mathscr{N}) / \tilde{\mathscr{F}}$ is isomorphic to $\mathscr{G} / \mathscr{N} \mathscr{F}$. Part (f) follows from [2], Proposition 2.4.

Lemma 5.2. - We assume that the orbit $\mathscr{O}_{1}$ is an affine variety over $k$. Then for each $m \in \mathbb{N}^{*}$, the orbit $\mathscr{O}_{m}$ is an affine variety over $k$.

Proof. - As we have a finite, surjective morphism $\mathscr{H}_{1} / \mathscr{C}_{1}^{0} \rightarrow \mathscr{H}_{1} / \mathscr{S}_{1} \stackrel{\sim}{\rightarrow} \mathscr{O}_{1}$, from our hypothesis we get that the quotient variety $\mathscr{H}_{1} / \mathscr{C}_{1}^{0}$ is affine. Thus $\mathscr{C}_{1}^{0}$ is exact in $\mathscr{H}_{1}$, cf. Theorem 5.1 (a).

Let $\mathscr{C}_{1, m}^{0}$ be the pullback of $\mathscr{C}_{1}^{0}$ via the reduction epimorphism $\mathscr{H}_{m} \rightarrow \mathscr{H}_{1}$. As $\operatorname{Ker}\left(\mathscr{H}_{m} \rightarrow \mathscr{H}_{1}\right)$ is a unipotent group (cf. [25], Lemma 2.2.3) which is smooth and connected and as $\mathscr{C}_{1}^{0}$ is a unipotent, smooth, connected group, we get that the group $\mathscr{C}_{1, m}^{0}$ is a unipotent, smooth, connected group. As $\mathscr{C}_{1}^{0}$ is exact in $\mathscr{H}_{1}$, from Theorem 5.1 (e) we get that $\mathscr{C}_{1, m}^{0}$ is exact in $\mathscr{H}_{m}$. The subgroup $\mathscr{C}_{m}^{0}$ of $\mathscr{C}_{1, m}^{0}$ is exact in $\mathscr{C}_{1, m}^{0}$, cf. Theorem 5.1 (c). From the last two sentences and Theorem 5.1 (d), we get that $\mathscr{C}_{m}^{0}$ is exact in $\mathscr{H}_{m}$. Thus the quotient variety $\mathscr{H}_{m} / \mathscr{C}_{m}^{0}$ is affine, cf. Theorem 5.1 (a).

As we have a finite, surjective morphism $\mathscr{H}_{m} / \mathscr{C}_{m}^{0} \rightarrow \mathscr{H}_{m} / \mathscr{S}_{m} \stackrel{\sim}{\rightarrow} \mathscr{O}_{m}$, we get from Chevalley's theorem that $\mathscr{O}_{m}$ is an affine variety over $k$. 


\subsection{Proof of Theorem 1.2}

Based on Subsection 2.3, we can assume that we are in the Essential Situation 1.9. In particular, $D_{m}=D\left[p^{m}\right]$ and $k$ is algebraically closed. We recall that we are assuming that the condition (C) holds for $D_{m}$ (see the introduction) and thus also for $D$. As $\mathfrak{s}_{D}(m)=\mathscr{A}_{D}(\mathscr{E})$ is equal to $\mathscr{A}_{D^{\vee}}(\mathscr{E} \vee)$, by replacing if needed $(D, \mathscr{E})$ with $\left(D^{\vee}, \mathscr{E}^{\vee}\right)$ we can assume that $\kappa(D)<1$. Therefore we can choose the permutation $\pi$ of Subsection 4.1 such that $\kappa(\pi)<1$. We also recall that $\tilde{\mathscr{C}}_{1}^{0}$ is a connected, smooth, unipotent subgroup of $\mathscr{I}_{1}$. As $\kappa(\pi)<1$, from Theorem 4.8 we get that we have a natural finite, flat morphism $\mathscr{V}_{2} \times_{k} \mathscr{W}_{0, k} \rightarrow \tilde{\mathscr{C}}_{1}^{0} \backslash \mathscr{I}_{1}$ of degree $p^{\left|\mathscr{J}_{0,0}\right|}$ (in the smooth, faithfully flat topology, this morphism looks like the morphism $\zeta$ ). From this and Chevalley's theorem, we get that the quotient variety $\tilde{\mathscr{C}}_{1}^{0} \backslash \mathscr{I}_{1}$ is affine. As the quotient varieties $\tilde{\mathscr{C}}_{1}^{0} \backslash \mathscr{I}_{1}$ and $\mathscr{I}_{1} / \tilde{\mathscr{C}}_{1}^{0}$ are isomorphic, we get that $\mathscr{I}_{1} / \tilde{\mathscr{C}}_{1}^{0}$ is affine. Thus $\tilde{\mathscr{C}}_{1}^{0}$ is an exact subgroup of $\mathscr{I}_{1}$, cf. Theorem 5.1 (a). Therefore the unipotent group $\mathscr{W}_{-, k} \mathscr{C}_{1}^{0}$ is an exact subgroup scheme of $\mathscr{H}_{1}$, cf. Theorem 5.1 (e). From this and Theorem 5.1 (c) and (d), we get that $\mathscr{C}_{1}^{0}$ is an exact subgroup of $\mathscr{H}_{1}$. As we have a finite, surjective morphism $\mathscr{H}_{1} / \mathscr{C}_{1}^{0} \rightarrow \mathscr{H}_{1} / \mathscr{S}_{1} \stackrel{\sim}{\rightarrow} \mathscr{O}_{1}$, from Theorem 5.1 and Chevalley's theorem, we get that $\mathscr{O}_{1}$ is affine. From this and Lemma 5.2, we get that:

COROLlary 5.3. - We assume that the condition (C) holds for D. Then for each $m \in \mathbb{N}^{*}$, the orbit $\mathscr{O}_{m}$ is an affine variety over $k$.

We can complete the proof of Theorem 1.2 as follows. Locally in the Zariski topology of $\mathscr{A}$, we can write $\mathscr{A}=\operatorname{Spec} A$ and we can assume that the evaluation of the Dieudonné crystal $\mathbb{D}(\mathscr{E})$ of $\mathscr{E}$ at the thickening $\mathscr{A} \hookrightarrow \operatorname{Spec} W(A)$ is, when viewed without connection and Verschiebung map, a pair of the form $\left(M \otimes_{W(k)} W(A), g_{A}\left(\phi \otimes \sigma_{A}\right)\right)$ with $g_{A} \in \mathbf{G L}_{M}(W(A))$. The reduction $g_{A}[m] \in \mathbf{G L}_{M}\left(W_{m}(A)\right)=\mathscr{D}_{m}(A)$ of $g_{A}$ is a morphism $\varpi: \mathscr{A} \rightarrow \mathscr{D}_{m}$. The locally closed embedding $\mathfrak{s}_{D}(m) \hookrightarrow \mathscr{A}$ is the pullback of the locally closed embedding $\mathscr{O}_{m} \hookrightarrow \mathscr{D}_{m}$ via $\varpi$ (cf. [25], Subsubsection 3.1.1).

From the previous paragraph and Corollary 5.3, we get that in general $\mathfrak{s}_{D}(m)$ is an affine $\mathscr{A}$-scheme. This ends the proof of Theorem 1.2.

REMARK 5.4. - Under the natural identification $\mathscr{I}_{1}=\mathscr{W}_{+0, k}$, the group $\tilde{\mathscr{C}}_{1}^{0}$ gets identified with the crystalline realization $\operatorname{Aut}(D[p])_{\text {crys,red }}^{0}$ of the identity component $\operatorname{Aut}(D[p])_{\text {red }}^{0}$ of the reduced group scheme of automorphisms of $D[p]$ (cf. [25], Theorem 2.4 (b)). Thus the quotient variety $\mathscr{W}_{+0, k} / \operatorname{Aut}(D[p])_{\text {crys,red }}^{0}$ is affine. It is easy to see that $\mathfrak{n}_{0,0} \oplus \mathfrak{n}_{+, 1}$ is the $k$-span of elements $g-1_{M}[1]$ with $g \in \operatorname{Aut}(D[p])_{\text {crys,red }}^{0}(k)=\operatorname{Aut}(D[p])_{\text {red }}^{0}(k)$. Therefore the number $\left|\mathscr{J}_{0,0}\right|$ is an invariant of the isomorphism class of $D[p]$.

\section{Level $m$ stratifications for quasi Shimura $p$-varieties of Hodge type}

In this section, we consider the relative version of the action $\mathbb{T}_{m}$. We prove Proposition 6.1, an analogue of Corollary 5.3, which will imply Theorem 1.6 and its analogues for general level $m$ stratifications.

$4^{\mathrm{e}}$ SÉRIE - TOME $43-2010-\mathrm{N}^{\mathrm{o}} 6$ 


\subsection{Orbits of relative group actions}

Let $G$ be a smooth, closed subgroup scheme of $\mathbf{G L}_{M}$ such that its generic fibre $G_{B(k)}$ is connected. Thus the scheme $G$ is integral. Until the end we will assume that the following two axioms hold for the triple $(M, \phi, G)$ :

(i) The Lie subalgebra $\operatorname{Lie}\left(G_{B(k)}\right)$ of $G_{B(k)}$ is stable under $\phi$, i.e. we have $\phi\left(\operatorname{Lie}\left(G_{B(k)}\right)\right)=\operatorname{Lie}\left(G_{B(k)}\right)$.

(ii) There exist a direct sum decomposition $M=F^{1} \oplus F^{0}$ and a smooth, closed subgroup scheme $G_{1}$ of $\mathbf{G L}_{M}$ such that the following four properties hold:

(a) The kernel of the reduction modulo $p$ of $\phi$ is $F^{1} / p F^{1}$.

(b) The cocharacter $\mu: \mathbb{G}_{m} \rightarrow \mathbf{G L}_{M}$ which fixes $F^{0}$ and acts via the inverse of the identical character of $\mathbb{G}_{m}$ on $F^{1}$, factors through $G_{1}$.

(c) The group scheme $G_{1}$ contains $G$ in such a way that we have a short exact sequence $1 \rightarrow G \rightarrow G_{1} \rightarrow \mathbb{G}_{m}^{u} \rightarrow 1$ with $u \in\{0,1\}$.

(d) If $u=1$ (i.e., if $G_{1} \neq G$ ), then the homomorphism $\mu: \mathbb{G}_{m} \rightarrow G_{1}$ defined by $\mu$ (cf. properties (b) and (c)) is a splitting of the short exact sequence of the property (c).

We will use the notations of Section 3 for the direct sum decomposition $M=F^{1} \oplus F^{0}$ of the axiom (ii). Due to the properties (ii.b) and (ii.c) we have a direct sum decomposition into $W(k)$-modules

$$
\operatorname{Lie}(G)=\oplus_{i=-1}^{1} \tilde{F}^{i}(\operatorname{Lie}(G))
$$

such that $\mu$ acts via inner conjugation on $\tilde{F}^{i}(\operatorname{Lie}(G))$ as the $-i$-th power of the identical character of $\mathbb{G}_{m}$. Let $e_{+}, e_{0}$, and $e_{-}$be the ranks of $\tilde{F}^{1}(\operatorname{Lie}(G)), \tilde{F}^{0}(\operatorname{Lie}(G))$, and $\tilde{F}^{-1}(\operatorname{Lie}(G))$ (respectively). Let $d_{G}:=\operatorname{dim}\left(G_{k}\right)=\operatorname{dim}\left(G_{B(k)}\right)$. Due to (17), we have

$$
d_{G}=e_{+}+e_{0}+e_{-} .
$$

We consider the following four closed subgroup schemes $\mathscr{W}_{+}^{G}:=\mathscr{W}_{+} \cap G, \mathscr{W}_{0}^{G}:=\mathscr{W}_{0} \cap G$, $\mathscr{W}_{-}^{G}:=\mathscr{W}_{-} \cap G$, and $\mathscr{W}_{+0}^{G}:=\mathscr{W}_{+0} \cap G$ of $G$. Let

$$
\mathscr{H}^{G}:=\mathscr{W}_{+}^{G} \times_{W(k)} \mathscr{W}_{0}^{G} \times_{W(k)} \mathscr{W}_{-}^{G}
$$

it is a closed subscheme of $\mathscr{H}$ such that $\mathscr{H}_{W_{m}(k)}^{G}$ is a closed subgroup subscheme of $\mathscr{H}_{W_{m}(k)}=\tilde{\mathscr{H}}_{W_{m}(k)}$ (we recall that we view the isomorphism $\mathscr{P}_{W_{m}(k)}: \mathscr{H}_{W_{m}(k)} \stackrel{\sim}{\rightarrow} \tilde{\mathscr{H}}_{W_{m}(k)}$ of Spec $W_{m}(k)$-schemes as a natural identification).

The group schemes $\mathscr{W}_{+}^{G}$ and $\mathscr{W}_{-}^{G}$ over Spec $W(k)$ are isomorphic to $\mathbb{G}_{a}^{e_{+}}$and $\mathbb{G}_{a}^{e_{-}}$ (respectively). More precisely, if $R$ is a commutative $W(k)$-algebra, then we have identities $\mathscr{W}_{+}^{G}(R)=1_{M \otimes_{W(k)} R}+\tilde{F}^{1}(\operatorname{Lie}(G)) \otimes_{W(k)} R$ and $\mathscr{W}_{-}^{G}(R)=1_{M \otimes_{W(k)} R}+\tilde{F}^{-1}(\operatorname{Lie}(G)) \otimes_{W(k)} R$.

Let $\mathscr{W}_{0}^{G_{1}}:=\mathscr{W}_{0} \cap G_{1}$. The group schemes $\mathscr{W}_{0}^{G_{1}}$ and $\mathscr{W}_{0}^{G}$ are smooth (see [25], Subsubsection 4.1.1).

The Lie algebras of $W_{+}^{G}, W_{0}^{G}$, and $W_{-}^{G}$ are $\tilde{F}^{1}(\operatorname{Lie}(G)), \tilde{F}^{0}(\operatorname{Lie}(G))$, and $\tilde{F}^{-1}(\operatorname{Lie}(G))$ (respectively). This implies that the relative dimension of $\mathscr{W}_{0}^{G}$ is $e_{0}$. The smooth, affine scheme $\mathscr{H}^{G}$ has relative dimension $d_{G}$ over Spec $W(k)$. The natural product morphism $\mathscr{P}_{0}^{G}: \mathscr{H}^{G} \rightarrow G$ is induced naturally by the open embedding $\mathscr{P}_{0}: \mathscr{H} \hookrightarrow \mathbf{G L}_{M}$ and therefore it is also an open embedding. Let $\mathscr{P}_{-}^{G}:=1_{\mathscr{W}_{+}^{G}} \times 1_{\mathscr{W}_{0}^{G}} \times p 1_{\mathscr{W}_{-}^{G}}: \mathscr{H}^{G} \rightarrow \mathscr{H}^{G}$. The composite morphism $\mathscr{P}_{0-}^{G}:=\mathscr{P}_{0}^{G} \circ \mathscr{P}_{-}^{G}: \mathscr{H}^{G} \rightarrow G$ has the property that its reduction 
$\mathscr{P}_{0-, W_{m}(k)}^{G}: \mathscr{H}_{W_{m}(k)}^{G} \rightarrow G_{W_{m}(k)}$ modulo $p^{m}$ is a homomorphism of affine group schemes over Spec $W_{m}(k)$ which is a restriction of the homomorphism $\mathscr{P}_{0-, W_{m}(k)}: \mathscr{H}_{W_{m}(k)} \rightarrow$ $\mathbf{G L}_{M / p^{m} M}$ (see Section 3 for $\mathscr{P}_{0-}$ ).

Let $\tilde{\mathscr{H}}^{G}$ be the dilatation of $G$ centered on the smooth subgroup $\mathscr{W}_{+0, k}^{G}$ of $G_{k}$; it is a smooth, closed subgroup scheme of $\tilde{\mathscr{H}}$. As in Section 3, we argue that we have a natural morphism $\mathscr{P}^{G}: \mathscr{H}^{G} \rightarrow \tilde{\mathscr{H}}^{G}$ of Spec $W(k)$-schemes which gives birth to an isomorphism $\mathscr{P}_{W_{m}(k)}^{G}: \mathscr{H}_{W_{m}(k)}^{G} \stackrel{\sim}{\rightarrow} \tilde{\mathscr{H}}_{W_{m}(k)}^{G}$ of Spec $W_{m}(k)$-schemes, to be viewed as an identification. The group schemes structures on $\mathscr{H}_{W_{m}(k)}^{G}$ induced via the identification $\mathscr{P}_{W_{m}(k)}^{G}$ or via the identification of $\mathscr{H}_{W_{m}(k)}^{G}$ with a closed subgroup scheme of $\mathscr{H}_{W_{m}(k)}$, are equal.

Let $\mathscr{H}_{m}^{G}:=\mathbb{W}_{m}\left(\mathscr{H}^{G}\right)=\mathbb{W}_{m}\left(\tilde{\mathscr{H}}^{G}\right)$; it is a smooth, affine group over $k$ of dimension $m d_{G}$ which is connected if and only if $\mathscr{H}_{k}^{G}$ (equivalently $\mathscr{W}_{0, k}^{G}$ ) is connected (cf. Section 3). Let $\mathscr{D}_{m}^{G}:=\mathbb{W}_{m}(G)$; it is a smooth, affine $k$-scheme of dimension $m d_{G}$ which is connected if and only if $G_{k}$ is connected (cf. Section 3). There exists a unique action

$$
\mathbb{T}_{m}^{G}: \mathscr{H}_{m}^{G} \times_{k} \mathscr{D}_{m}^{G} \rightarrow \mathscr{D}_{m}^{G}
$$

which is the natural restriction of the action $\mathbb{T}_{m}$ of Section 3.

Let $\mathscr{O}_{m}^{G}$ be the orbit of $1_{M}[m] \in \mathscr{D}_{m}^{G}(k)$ under the action $\mathbb{T}_{m}^{G}$. Let $\mathscr{S}_{m}^{G}$ be the stabilizer subgroup scheme of the point $1_{M}[m] \in \mathscr{D}_{m}^{G}(k)$ under the action $\mathbb{T}_{m}^{G}$; we have $\mathscr{S}_{m}^{G}:=\mathscr{S}_{m} \cap \mathscr{H}_{m}^{G}$. Let $\mathscr{C}_{m}^{G}$ be the reduced group of $\mathscr{S}_{m}^{G}$ and let $\mathscr{C}_{m}^{0 G}$ be the identity component of $\mathscr{C}_{m}^{G}$.

Proposition 6.1. - If the condition (C) holds for D, then the orbit $\mathscr{O}_{m}^{G}$ of $1_{M}[m] \in \mathscr{D}_{m}^{G}(k)$ under the action $\mathbb{T}_{m}^{G}$ is a smooth, affine $k$-scheme. Therefore, if either $p=3$ and $\min \{c, d\} \leq 6$ or $p \geq 5$, then each orbit of the action $\mathbb{T}_{m}^{G}$ is a smooth, affine $k$-scheme.

Proof. - As $\mathscr{C}_{m}^{0}$ is a unipotent group, $\mathscr{C}_{m}^{0 G}$ is exact in $\mathscr{C}_{m}^{0}$ (cf. Theorem 5.1 (c)). From this and the fact that $\mathscr{C}_{m}^{0}$ is exact in $\mathscr{H}_{m}$ (cf. Subsection 5.2), we get that $\mathscr{C}_{m}^{0 G}$ is exact in $\mathscr{H}_{m}$ (cf. Theorem 5.1 (d)) and thus also in $\mathscr{H}_{m}^{G}$ (cf. Theorem 5.1 (f)). As in Subsection 5.2, we argue that this implies that $\mathscr{O}_{m}^{G}$ is a smooth, affine $k$-scheme.

ExAmple 6.2. - Let $a \geq 2$ be an integer. We assume that we have a direct sum decomposition $M=\oplus_{s=1}^{a} M_{s}$ such that $F^{1} \subseteq M_{1}$ and for all $s \in\{1, \ldots, a\}$ we have $\phi\left(M_{s}\right) \subseteq M_{s+1}$ (here $\left.M_{a+1}:=M_{1}\right)$. We identify naturally $W\left(\mathbb{F}_{p^{a}}\right)$ with a $\mathbb{Z}_{p}$-subalgebra of $\operatorname{End}(M, \phi)$ (equivalently of $\operatorname{End}(D)$ ) that acts on each $M_{s}$ via scalar endomorphisms. From Example 4.4, we get that the condition (C) holds for $D$. Thus $\mathscr{O}_{m}^{G}$ is a smooth, affine $k$-scheme, cf. Proposition 6.1. Thus, if $G$ is a closed subgroup scheme of $\prod_{s=1}^{a} \mathbf{G L}_{M_{s}}$, then each orbit of $\mathbb{T}_{m}^{G}$ is a smooth, affine $k$-scheme. We emphasize that these hold for all primes $p$.

\subsection{Quasi Shimura $p$-varieties of Hodge type}

In this subsection, we assume that $c=d$ and we use only $d$. We also assume that the condition $(\mathrm{C})$ holds for each $p$-divisible group over $k$ which admits principal quasipolarizations and has dimension $d$ (e.g., if $p \geq 5$ ). Suppose that $D$ has a principal quasipolarization $\lambda$. Let $\psi: M \times M \rightarrow W(k)$ be the perfect, alternating form on $M$ induced naturally by $\lambda$; for $x, y \in M$, we have $\psi(\phi(x), \phi(y))=p \sigma(\psi(x, y))$. Suppose that $G$ is a closed subgroup scheme of $\mathbf{S p}(M, \psi)$. We recall that axioms (i) and (ii) of Subsection 6.1 
hold for the triple $(M, \phi, G)$. As $\mu: \mathbb{G}_{m} \rightarrow G_{1}$ cannot factor through $\mathbf{S p}(M, \psi)$, we have $u=1$ (i.e., we have a short exact sequence $1 \rightarrow G \rightarrow G_{1} \rightarrow \mathbb{G}_{m} \rightarrow 1$ ). Let

$$
\mathscr{F}:=\{(M, g \phi, \psi, G) \mid g \in G(W(k))\} ;
$$

it is a family of principally quasi-polarized Dieudonné modules with a group over $k$. Let $\mathscr{A}_{d, 1, N}$ be as in the introduction.

Let $\mathscr{M}$ be a quasi Shimura $p$-variety of Hodge type relative to $\mathscr{F}$ in the sense of [25], Definition 4.2.1. Thus $\mathscr{M}$ is a smooth $k$-scheme which is equidimensional and which is equipped with a morphism $\mathscr{M} \rightarrow \mathscr{A}_{d, 1, N, k}$ that induces $k$-epimorphisms at the level of complete, local rings of residue field $k$ (i.e., it is a formal closed embedding at all $k$-valued points) and that satisfies an extra axiom that involves $\mathscr{F}$ (see [25], Axiom 4.2.1 (iii)). This extra axiom implies that $\mathscr{M}$ has a level $m$ stratification $\mathscr{S}^{G}(m)$ in the sense of [24], Definition 2.1.1. For each algebraically closed field $K$ that contains $k$, we have a set $\mathscr{S}_{m}^{G}(K)$ of reduced locally closed subschemes of $\mathscr{M}_{K}$ which are regular, equidimensional and which locally in the étale topology of $\mathscr{M}_{K}$ are pullbacks of locally closed embeddings of the form $\mathscr{O}^{G} \hookrightarrow \mathscr{H}_{m, K}^{G}$ for some orbit $\mathscr{O}^{G}$ of the extension of the action $\mathbb{T}_{m}^{G}$ to $K$ (cf. [25], Subsubsection 4.2.3). From this and the analogue of the Proposition 6.1 over $K$, we get that each element of $\mathscr{S}_{m}^{G}(K)$ is pure in $\mathscr{M}_{K}$. Thus we get that:

Theorem 6.3. - The level $m$ stratification $\mathscr{S}_{m}^{G}$ of $\mathscr{M}$ is pure. In other words, for each algebraically closed field $K$ that contains $k$, all the elements of $\mathscr{S}_{m}^{G}(K)$ (i.e., all strata of $\mathscr{S}_{m}^{G}$ which are subschemes of $\mathscr{M}_{K}$ ) are affine $\mathscr{M}_{K}$-schemes (equivalently, are affine $\mathscr{M}$-schemes).

COROLlary 6.4. - Let $K$ be an algebraically closed field extension of $k$. Let $\mathfrak{s} \in \mathscr{S}_{m}^{G}(K)$. Let $\overline{\mathfrak{s}}$ be the Zariski closure of $\mathfrak{s}$ in $\mathscr{M}_{K}$. Then the reduced scheme underlying $\overline{\mathfrak{s}} \backslash \mathfrak{s}$ is either empty or of pure codimension 1 in $\overline{\mathfrak{s}}$.

ExAmple 6.5. - Suppose that $G=\mathbf{S p}\left(M, \psi_{M}\right)$ and $G_{1}=\mathbf{G S p}\left(M, \psi_{M}\right)$. Then $\mathscr{A}_{d, 1, N, k}$ is a quasi Shimura $p$-variety of Hodge type relative to $\mathscr{F}$, cf. [25], Example 4.5. The strata of $\mathscr{S}_{m}^{G}(k)$ are of the form $\mathfrak{s}_{D, \lambda}(m)$, cf. loc. cit. Thus Theorem 1.6 is a particular case of Theorem 6.3.

Remark 6.6. - One can use Example 6.2 to get plenty of examples of level $m$ stratifications in characteristic 2 or 3 which are pure.

\section{On principal purity for stratifications}

In Definition 1.7, we introduced the stronger notion for a subscheme to be principally pure (with respect to some Grothendieck topology). In this section, we investigate the question whether the $p$-rank stratification is principally pure. This is connected to the existence of generalized Hasse-Witt invariants, i.e. to the local existence of functions $f$ such that a stratum is defined in its scheme-theoretic closure as the locus where $f$ is invertible.

In [10], Theorem on p. 1567, T. Itō proves the existence of generalized Hasse-Witt invariants for $p$-rank strata of the special fibre (at a split prime $p$ ) of a good integral model of a unitary Shimura variety $\operatorname{Sh}(\mathscr{G}, \mathscr{X})$ which is of PEL type and which over $\mathbb{R}$ involves a derived group $\mathscr{G}_{\mathbb{R}}^{\text {der }}=\mathbf{S U}(n-1,1) \times \mathbf{S U}(n, 0) \times \cdots \times \mathbf{S U}(n, 0)$. In this case, the $p$-rank strata 
coincide with the level $m$ strata for all $m$. He derives the corollary that the $p$-rank strata are affine. From the affineness result and the weak Lefschetz theorem, he obtains that the number of connected components of all Zariski closures of positive dimensional $p$-rank strata are equal.

In general, however, principal purity (for the étale topology) does not hold for $p$-rank strata, even over regular schemes, as the following example shows.

Example 7.1. - We set $\quad c=d=2$. Let $\mathscr{R}:=k \llbracket x_{1}, x_{2}, x_{3}, x_{4} \rrbracket \quad$ and $R:=\mathscr{R} /\left(x_{1} x_{4}-x_{2} x_{3}\right)$. Let $S:=\operatorname{Spec} \mathscr{R}$ and $T:=\operatorname{Spec} R$. Let $g$ be the $\mathscr{R}$-linear automorphism of $N:=\mathscr{R}^{4}$ associated to an invertible block matrix

$$
\mathbf{A}=\left(\begin{array}{ll}
A_{1} & A_{2} \\
A_{3} & A_{4}
\end{array}\right)
$$

formed by $2 \times 2$ blocks, where

$$
A_{1}=\left(\begin{array}{ll}
x_{1} & x_{2} \\
x_{3} & x_{4}
\end{array}\right) .
$$

Let $\left\{\bar{e}_{1}, \bar{e}_{2}, \bar{e}_{3}, \bar{e}_{4}\right\}$ be the standard basis for $N$. Let $\phi_{N}: N \otimes_{\mathscr{R}, \sigma_{\mathscr{R}}} \mathscr{R} \rightarrow N$ be the $\mathscr{R}$-linear map that takes the quadruple $\left(\bar{e}_{1} \otimes 1, \bar{e}_{2} \otimes 1, \bar{e}_{3} \otimes 1, \bar{e}_{4} \otimes 1\right)$ to the quadruple $\left(g\left(\bar{e}_{1}\right), g\left(\bar{e}_{2}\right), 0,0\right)$. Let $\vartheta_{N}: N \rightarrow N \otimes_{\mathscr{R}, \sigma_{\mathscr{R}}} \mathscr{R}$ be the $\mathscr{R}$-linear map that takes the quadruple $\left(g\left(\bar{e}_{1}\right), g\left(\bar{e}_{2}\right), g\left(\bar{e}_{3}\right), g\left(\bar{e}_{4}\right)\right)$ to the quadruple $\left(0,0, \bar{e}_{3} \otimes 1, \bar{e}_{4} \otimes 1\right)$. It is easy to see that the triple $\left(N, \phi_{N}, \vartheta_{N}\right)$ is the Dieudonné crystalline functor (viewed without connection) of a $B T_{1}$ over $S$, to be denoted as $B$.

One computes the $p$-rank of $B$ at a geometric point $x$ of $S$ as follows. Let $N_{1}$ be the direct summand of $N$ generated by $\bar{e}_{3}$ and $\bar{e}_{4}$. The kernel of $\phi_{N}$ is $N_{1} \otimes_{\mathscr{R}, \sigma \mathscr{R}} \mathscr{R}$. Let $\bar{\phi}_{N}:\left(N / N_{1}\right) \otimes_{\mathscr{R}, \sigma_{\mathscr{R}}} \mathscr{R} \rightarrow N / N_{1}$ be the $\mathscr{R}$-linear map induced naturally by $\phi_{N}$. We view $\left\{\bar{e}_{1}, \bar{e}_{2}\right\}$ as an $\mathscr{R}$-basis for $N / N_{1}$. One looks for solutions of the equation $\bar{\phi}_{N}\left(\left(z_{1} \bar{e}_{1}+z_{2} \bar{e}_{2}\right) \otimes 1\right)=z_{1} \bar{e}_{1}+z_{2} \bar{e}_{2}$. One comes across the following system $\mathscr{Y}$ of two equations

$$
z_{1}=x_{1} z_{1}^{p}+x_{2} z_{2}^{p}, \quad z_{2}=x_{3} z_{1}^{p}+x_{4} z_{2}^{p} .
$$

The $p$-rank of $B$ at $x$ is the dimension of the $\mathbb{F}_{p}$-vector space of solutions of $\mathscr{Y}$ at $x$. Therefore the $p$-rank stratification of $S$ has an open dense stratum $S \backslash T$ (of $p$-rank 2) and has one stratum $T \backslash Y$ of codimension 1 in $S$ (of $p$-rank 1). Here $Y$ is the smallest (thus reduced) closed subscheme of $T$ with the property that $\mathscr{Y}$ defines a scheme over $T$ which is an étale cover of degree $p$ above $T \backslash Y$.

We show that the assumption that $Y$ is the zero locus of a single function $f \in R$ leads to a contradiction. To solve $\mathscr{Y}$ over $T$, we remark that since we have $x_{1} x_{4}=x_{2} x_{3}$ in $R$, we also have $x_{3} z_{1}=x_{1} z_{2}$. Thus to solve $\mathscr{Y}$ over $T^{\prime}:=\operatorname{Spec} R\left[\frac{1}{x_{1}}\right]$, we can substitute $z_{2}=x_{1}^{-1} x_{3} z_{1}$ into the first equation of $\mathscr{Y}$ and get the equation $z_{1}=\left(x_{1}+x_{1}^{-p} x_{2} x_{3}^{p}\right) z_{1}^{p}$. One concludes that $Y \cap T^{\prime}$ is the zero locus of the function $x_{1}+x_{1}^{-p} x_{2} x_{3}^{p}=x_{1}^{1-p}\left(x_{1}^{p}+x_{4} x_{3}^{p-1}\right)$ in $T^{\prime}$. One checks that $T^{\prime}$ is regular and that $\operatorname{Spec} R\left[\frac{1}{x_{1}}\right] /\left(x_{1}^{p}+x_{4} x_{3}^{p-1}\right)$ is regular and irreducible (irreducibility can be checked starting from the fact that $k\left[x_{1}, x_{2}, x_{3}, x_{4}\right] /\left(x_{1} x_{4}-x_{2} x_{3}, x_{1}^{p}+x_{4} x_{3}^{p-1}\right)\left[\frac{1}{x_{1}}\right]$ is isomorphic to $\left.k\left[x_{1}, x_{3}\right]\left[\frac{1}{x_{1} x_{3}}\right]\right)$. Moreover, each unit in $R\left[\frac{1}{x_{1}}\right]$ is a unit of $R$ times an integral power of $x_{1}$. From the last two sentences we get that the image of $f$ in $R\left[\frac{1}{x_{1}}\right]$ is equal to 
$\left(x_{1}^{p}+x_{4} x_{3}^{p-1}\right)^{v} x_{1}^{t} u$, where $v$ is a positive integer, where $t$ is an integer, and where $u$ is a unit in $R$. It is easy to see that $t \geq 0$ and therefore the equations $x_{1}=x_{3}=x_{2}=0$ define a closed subscheme of $Y$. But over the locus defined by $x_{4} \neq 0$ and $x_{1}=x_{2}=x_{3}=0$, the $p$-rank of $B$ is 1 , and this is a contradiction to the $p$-rank being 0 there. Therefore, principal purity fails for the $p$-rank 1 stratum $T \backslash Y$ of $S$.

\subsection{Proof of Proposition 1.8}

Let $S$ and $B$ be as in Example 7.1. From Example 7.1, we get that Proposition 1.8 holds for $c=d=2$ and $s=1$. Using direct sums of $B$ and of constant $B T_{1}$ 's over $S$, one easily gets that Proposition 1.8 holds for all $c, d \geq 2$ and $s \in\{1, \ldots, c-1\}$.

\section{Acknowledgments}

The first author was supported by a postdoctoral research fellowship of the FQRNT while enjoying the hospitality of the Institut de mathématiques de Jussieu (Université Paris 7); he thanks $\mathrm{O}$. Brinon for kindly sharing his $\mathrm{T}_{\mathrm{E}} \mathrm{X}$ expertise. The second author would like to thank Binghamton University for good working conditions; he was partially supported by the NSF grant \#0900967.

\section{REFERENCES}

[1] S. Bosch, W. Lütkebohmert, M. Raynaud, Néron models, Ergebn. Math. Grenzg. 21, Springer, 1990.

[2] E. Cline, B. Parshall, L. Scott, Induced modules and affine quotients, Math. Ann. 230 (1977), 1-14.

[3] P. Deligne, Variétés de Shimura: interprétation modulaire, et techniques de construction de modèles canoniques, in Automorphic forms, representations and L-functions (Proc. Sympos. Pure Math., Oregon State Univ., Corvallis, Ore., 1977), Part 2, Proc. Sympos. Pure Math., XXXIII, Amer. Math. Soc., 1979, 247-289.

[4] E. Z. Goren, Hasse invariants for Hilbert modular varieties, Israel J. Math. 122 (2001), $157-174$.

[5] A. Grothendieck, Éléments de Géométrie Algébrique. II. Étude globale élémentaire de quelques classes de morphismes, Publ. Math. I.H.É.S. 8 (1961), 222.

[6] A. Grothendieck, Éléments de Géométrie Algébrique. IV. Étude locale des schémas et des morphismes de schémas. II, Publ. Math. I.H.É.S. 24 (1965), 231.

[7] A. Grothendieck, J. Dieudonné, Éléments de Géométrie Algébrique, I. Le langage des schémas, Grundl. der Math. Wiss. 166, Springer, 1971. 
[8] G. Harder, Über die Galoiskohomologie halbeinfacher Matrizengruppen. II, Math. Z. 92 (1966), 396-415.

[9] L. Illusie, Déformations de groupes de Barsotti-Tate (d'après A. Grothendieck), Séminaire sur les pinceaux arithmétiques : la conjecture de Mordell (Paris, 1983/84), Astérisque 127 (1985), 151-198.

[10] T. Iто̄, Hasse invariants for some unitary Shimura varieties, in Algebraische Zahlentheorie, Mathematisches Forschungsinstitut Oberwolfach, 2005, 1565-1568.

[11] A. J. De Jong, F. Oort, Purity of the stratification by Newton polygons, J. Amer. Math. Soc. 13 (2000), 209-241.

[12] R. E. Kotтwitz, Points on some Shimura varieties over finite fields, J. Amer. Math. Soc. 5 (1992), 373-444.

[13] H. Kraft, Kommutative algebraische $p$-Gruppen (mit Anwendungen auf $p$-divisible Gruppen und abelschen Varietäten), preprint, 86 p., Univ. Bonn, 1975.

[14] G. Laumon, L. Moret-Bailly, Champs algébriques, Ergebn. Math. Grenzg. 39, Springer, 2000.

[15] Y. Manin, The theory of commutative formal groups in finite caracteristic, Russian Math. Surv. 18 (1963), 1-83.

[16] B. Moonen, Group schemes with additional structures and Weyl group cosets, in Moduli of abelian varieties (Texel Island, 1999), Progr. Math. 195, Birkhäuser, 2001, 255-298.

[17] B. Moonen, T. Wedhorn, Discrete invariants of varieties in positive characteristic, Int. Math. Res. Not. 2004 (2004), 3855-3903.

[18] D. Mumford, J. Fogarty, F. Kirwan, Geometric invariant theory, third ed., Ergebn. Math. Grenzg. 34, Springer, 1994.

[19] F. OORT, Newton polygons and formal groups: conjectures by Manin and Grothendieck, Ann. of Math. 152 (2000), 183-206.

[20] F. Oort, A stratification of a moduli space of abelian varieties, in Moduli of abelian varieties (Texel Island, 1999), Progr. Math. 195, Birkhäuser, 2001, 345-416.

[21] B. Pascal, Monodromie du faisceau pervers des cycles évanescents de quelques variétés de Shimura simples, Invent. Math. 177 (2009), 239-280.

[22] C. Traverso, $p$-divisible groups over fields, in Symposia Mathematica, Vol. XI (Convegno di Algebra Commutativa, INDAM, Rome, 1971), Academic Press, 1973, 45-65.

[23] C. Traverso, Specializations of Barsotti-Tate groups, in Symposia Mathematica, Vol. XXIV (Sympos., INDAM, Rome, 1979), Academic Press, 1981, 1-21.

[24] A. Vasiu, Crystalline boundedness principle, Ann. Sci. École Norm. Sup. 39 (2006), 245-300.

[25] A. VAsiu, Level $m$ stratifications of versal deformations of $p$-divisible groups, J. Algebraic Geom. 17 (2008), 599-641.

[26] A. VAsiu, Mod $p$ classification of Shimura F-crystals, Math. Nachr. 283 (2010), 10681113.

[27] A. VAsiu, Manin problems for Shimura varieties of Hodge type, preprint, arXiv:math/0209410.

$4^{\mathrm{e}}$ SÉRIE - TOME $43-2010-\mathrm{N}^{\mathrm{o}} 6$ 
[28] T. Wedhorn, The dimension of Oort strata of Shimura varieties of PEL-type, in Moduli of abelian varieties (Texel Island, 1999), Progr. Math. 195, Birkhäuser, 2001, 441-471.

[29] T. ZINK, On the slope filtration, Duke Math. J. 109 (2001), 79-95.

(Manuscrit reçu le 12 novembre 2008; accepté, après révision, le 18 janvier 2010.)

\author{
Marc-Hubert NicOLE \\ Institut mathématique de Luminy \\ Campus de Luminy \\ Case 907 \\ 13288 Marseille cedex 9, France \\ E-mail: nicole@iml.univ-mrs.fr \\ Adrian VAsiu \\ Department of Mathematical Sciences \\ Binghamton University \\ Binghamton, NY 13902-6000, U.S.A. \\ E-mail: adrian@math.binghamton.edu \\ Torsten WEDHORN \\ Universität Paderborn \\ Institut für Mathematik \\ Warburger Str. 100 \\ 33098 Paderborn, Germany \\ E-mail: wedhorn@math.uni-paderborn.de
}

